\title{
AN X-RAY SURVEY OF THE YOUNG STELLAR POPULATION OF THE LYNDS 1641 AND IOTA ORIONIS REGIONS
}

\author{
I. Pillitteri ${ }^{1}$, S. J. Wolk ${ }^{1}$, S. T. Megeath ${ }^{2}$, L. Allen ${ }^{3}$, J. Bally ${ }^{4}$, M. Gagné ${ }^{5}$, R. A. Gutermuth ${ }^{6}$, L. Hartman $^{7}$, \\ G. Micela ${ }^{8}$, P. Myers ${ }^{1}$, J. M. Oliveira ${ }^{9}$, S. Sciortino $^{8}$, F. Walter ${ }^{1}$, L. Rebull ${ }^{10}$, And J. Stauffer ${ }^{10}$ \\ ${ }^{1}$ SAO-Harvard Center for Astrophysics, Cambridge, MA 02138, USA; ipillitteri@cfa.harvard.edu \\ ${ }^{2}$ Department of Physics and Astronomy, University of Toledo, Toledo, OH 43606, USA \\ ${ }^{3}$ National Optical Astronomy Observatory, Tucson, AZ 85719, USA \\ ${ }^{4}$ University of Colorado, Boulder, CO 80309, USA \\ ${ }^{5}$ Department of Geology and Astronomy, West Chester University, West Chester, PA 19383, USA \\ ${ }^{6}$ Department of Astronomy, University of Massachusetts, Amherst, MA 01003, USA \\ ${ }^{7}$ University of Michigan, Ann Arbor, MI 48109, USA \\ ${ }^{8}$ INAF-Osservatorio Astronomico di Palermo, I-90134 Palermo, Italy \\ ${ }^{9}$ School of Physical and Geographical Sciences, Lennard-Jones Laboratories, Keele University, Staffordshire ST5 5BG, UK \\ ${ }^{10}$ CALTECH, Pasadena, CA 91125, USA \\ Received 2012 October 24; accepted 2013 March 15; published 2013 April 18
}

\begin{abstract}
We present an XMM-Newton survey of the part of the Orion A cloud south of the Orion Nebula. This survey includes the Lynds 1641 (L1641) dark cloud, a region of the Orion A cloud with very few massive stars and hence a relatively low ambient UV flux, and the region around the O9 III star $\iota$ Orionis. In addition to proprietary data, we used archival XMM data of the Orion Nebula Cluster (ONC) to extend our analysis to a major fraction of the Orion A cloud. We have detected 1060 X-ray sources in L1641 and the $\iota$ Ori region. About $94 \%$ of the sources have Two Micron All Sky Survey and Spitzer counterparts, 204 and 23 being Class II and Class I or protostar objects, respectively. In addition, we have identified 489 X-ray sources as counterparts to Class III candidates, given they are bright in X-rays and appear as normal photospheres at mid-IR wavelengths. The remaining 205 X-ray sources are likely distant active galactic nuclei or other galactic sources not related to Orion A. We find that Class III candidates appear more concentrated in two main clusters in L1641. The first cluster of Class III stars is found toward the northern part of L1641, concentrated around $\iota$ Ori. The stars in this cluster are more evolved than those in the Orion Nebula. We estimate a distance of 300-320 pc for this cluster showing that it is in the foreground of the Orion A cloud. Another cluster rich in Class III stars is located in L1641 South and appears to be a slightly older cluster embedded in the Orion A cloud. Furthermore, other evolved Class III stars are found north of the ONC toward NGC 1977.
\end{abstract}

Key words: stars: formation - stars: individual (Orion A, L1641, Iota Orionis, V380 Ori, V883 Ori)

Online-only material: color figures, machine-readable tables

\section{INTRODUCTION}

Stars form by the fragmentation and collapse of dense gas within molecular clouds (Carpenter 2000; O’Dell 2001; Allen et al. 2007; Bally 2008). Surveys of the population of young stars within a cloud have a crucial role in determining the history and the efficiency of star formation. A multi-wavelength approach is invaluable for this purpose because of the intense X-ray and IR radiation typical of young stars.

The first phases of stellar formation are characterized by strong infrared (IR) and X-ray band emission. The IR excess comes from the circumstellar disk and the inner envelope that surround the protostellar core (Class $0-\mathrm{I}$ and flat-spectrum objects). The disk is present in the subsequent stages (Class II objects or classical T Tauri stars, CTTS) until accretion ends and gas-rich disks are dissipated by radiation or stellar winds (Class III objects or weak T Tauri stars, WTTS) or eventually condense into planetesimals. X-rays are thought to come from a scaled-up version of the solar corona, which should form quite early in the pre-main-sequence (PMS) phase (Favata \& Micela 2003; Feigelson \& Montmerle 1999). In addition, a soft $\mathrm{X}$-ray component can arise from shocks formed by the accretion process or the interaction of outflows and the circumstellar material (Favata et al. 2005; Güdel et al. 2008; Brickhouse et al. 2010; Argiroffi et al. 2011). X-rays are thus a powerful tracer of youth, allowing us to identify young stars after their IR signatures have disappeared. Multi-wavelength surveys can map the distribution of stars as a function of the evolutionary class, thus enabling studies of the star formation history within molecular clouds.

One of the best-studied star-forming regions is the Orion molecular cloud (OMC) complex, located in the Orion OB1 association. Comprehensive overviews of the morphology of the OMC and the mechanisms that have shaped its complex star formation history are given by Bally (2008). Briefly, within the $\mathrm{OMC}$, two main giant clouds are identified, Orion A and Orion $\mathrm{B}$, extended in declination in the range roughly from $-10 \mathrm{deg}$ to $-5 \mathrm{deg}$ (Orion A) and from $-4 \mathrm{deg}$ to $+4 \mathrm{deg}$ (Orion B). Furthermore, several OB associations are present and their subgroups span an age range between $<1 \mathrm{Myr}$ and $10-12 \mathrm{Myr}$, for a total population estimated between 5000 and 20,000 stars.

We have focused our attention on the southern part of the $\mathrm{OMC}$ and the Orion A cloud, and in particular the region south of the Orion Nebula Cluster (ONC) that includes the gas filaments of Lynds 1641 North and South (L1641 N, L1641 S). This region also contains $\iota$ Orionis and the cluster NGC 1980 loosely associated with it. ८ Ori seems to be closer than L1641 and 

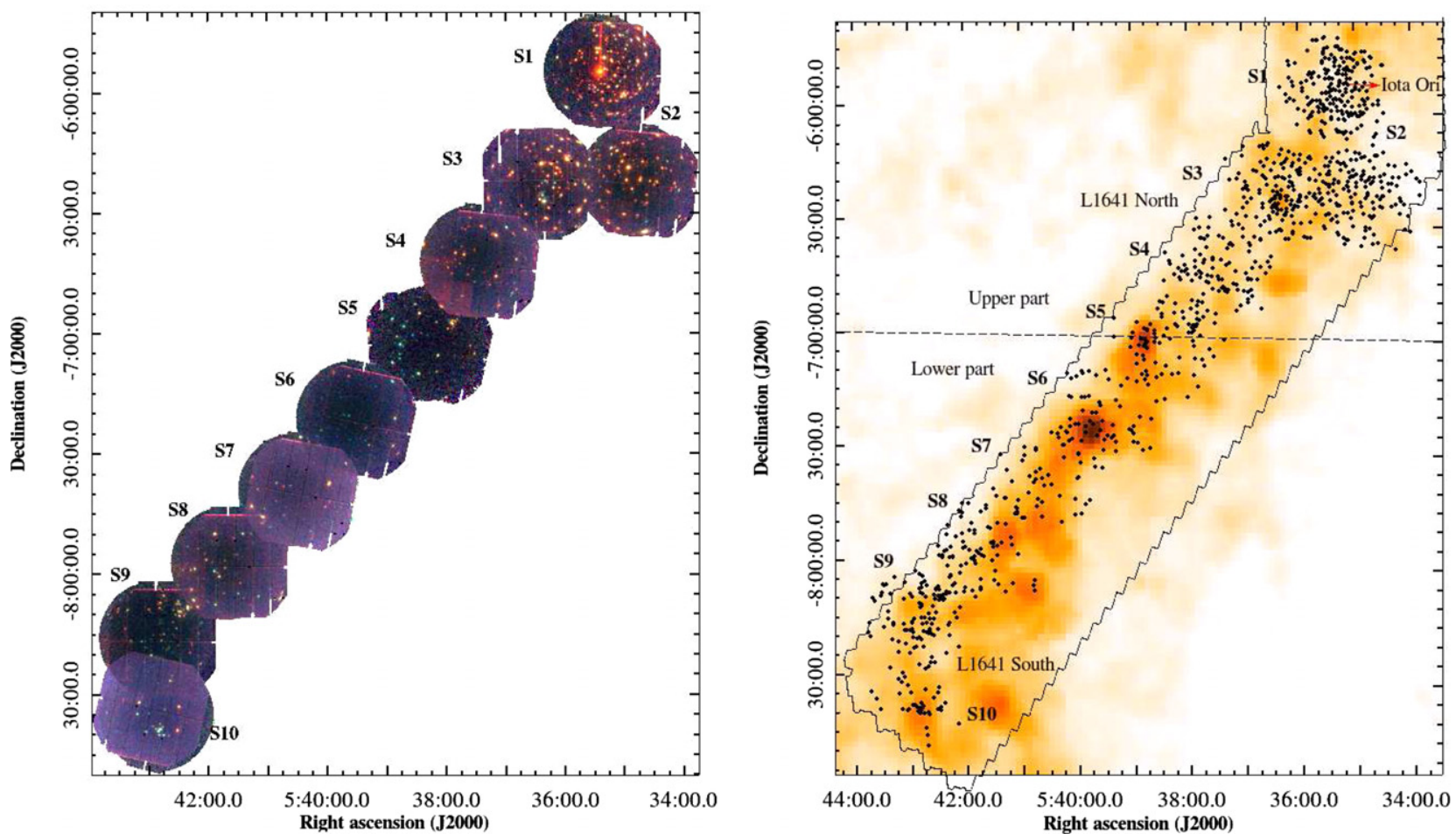

Figure 1. Left panel: RGB color image of the mosaic of SOXS fields. Bands used: red $=0.3-1.0 \mathrm{keV}$; green $=1.0-2.5$; blue $=2.5-8.0 \mathrm{keV}$. The fields are labeled Right panel: $A_{V}$ extinction map from Gutermuth et al. (2011) with the positions of X-ray sources. The scale is linear with darker colors indicating higher $A_{V}$ values. The range of $A_{V}$ is the same as in Figure 5, approximately from $\sim 1$ to $\simeq 18 \mathrm{mag}$. The black lines show the extent of the Spitzer field. About $60 \%$ of the Spitzer field of view has been observed by XMM-Newton, however, most of the YSOs are found in the densest part of the cloud.

(A color version of this figure is available in the online journal.)

the ONC by $\sim 10-50$ pc (Bally 2008) because of the absence of any reflection effect on the background cloud. The Orion A molecular cloud is less dense in this region than in the ONC. It has a filamentary shape approximately $0.5 \times 2.5$ long (see Figure 1). Maps in ${ }^{12} \mathrm{CO}$ and ${ }^{13} \mathrm{CO}$ reveal several dense, small clumps along the filament that host small groups of young stars (Bally et al. 1987; Strom et al. 1989; Allen 1995). A gradient of velocity in the gas cloud is detected from CO maps (Bally et al. 1987) that could imply a tilt to the cloud and a gradient in its distance decreasing from south to north, with the southern part being closer by $\leqslant 10 \%$. In this paper we will assume the distance to the ONC given by Menten et al. (2007; 414 pc).

X-ray surveys of L1641, obtained with Einstein (Strom et al. 1990), dramatically increased the number of known young stellar objects (YSOs) compared to previous optical/IR surveys. More recent observations with XMM-Newton and Chandra have studied only specific objects, like HH1/HH2 objects (with Chandra), and V883 Ori, V380 Ori (with XMM-Newton). Some of the XMM-Newton archival observations have been taken into account and reanalyzed in this paper to complete our survey (see Table 1). With the advent of Spitzer and its high sensitivity in the mid-IR, our knowledge of this region has been substantially enhanced (Megeath et al. 2012; Fang et al. 2009).

In contrast to the ONC, L1641 does not contain any early B type or O stars (Hsu et al. 2013; Allen et al. 2007). As a consequence, the strong ultraviolet (UV) flux illuminating the circumstellar disks and the envelopes of PMS stars of the ONC is almost absent in this region. The lack of strong UV and far-UV (FUV) fluxes may be important for the lifetime of disks, their evaporation, chemistry, and, in turn, the evolution of the angular momentum of stars, their activity, and, finally, the formation of planets around these stars (Guarcello et al. 2010; Wright et al. 2012).
The first step for addressing these issues is to have a complete census of the stars at different stages of their early evolution. For this purpose, we embarked on a Survey of Orion A with XMMNewton and Spitzer (SOXS). The goal of SOXS is to detect the relatively bright X-ray sources in the Orion A cloud and complete the census of more evolved YSOs in this region. These stars represent a sample of cloud members chosen with minimal a priori bias toward their IR properties. We can then examine the IR and spatial properties of these sources to ascertain the distribution of YSOs within the cloud. Figure 1 (right panel) shows a map of extinction, overlaid on the Spitzer sky coverage and labels to indicate the different regions that we will discuss in the paper. The XMM-Newton survey covers about $60 \%$ of the sky coverage of the Spitzer survey, although it comprises the majority of the groups of YSOs detected in IR. This paper presents the catalog of sources detected in the X-ray survey of SOXS and their basic X-ray characteristic, as well as clustering metrics of the young stellar population in L1641. The structure of the paper is as follows. In Section 2 we describe the data we have acquired and their analysis, in Sections 3 and 4 we report our results, in Section 5 we discuss the discovery of new groups of Class III stars, and in Section 6 we give our conclusions.

\section{OBSERVATIONS AND DATA ANALYSIS}

The SOXS survey is composed of seven specifically proposed $\sim 50 \mathrm{ks} X M M$-Newton fields, south of the ONC, to which we added four archival fields in the same region. The archival fields are centered on $\iota$ Orionis, V380 Ori, and V883 Ori, respectively (Figure 1). As shown in Figure 1 (right panel), we have observed in X-rays the dense part of the filament of L1641 while Spitzer has observed a wider area (Megeath et al. 2012; Gutermuth et al. 2011). 
Table 1

List of XMM-Newton Observations

\begin{tabular}{|c|c|c|c|c|c|c|c|}
\hline \multicolumn{8}{|c|}{ Southern Fields } \\
\hline Field Name & ObsID & $\begin{array}{c}\text { R.A. } \\
(\mathrm{J} 2000)\end{array}$ & $\begin{array}{c}\text { Decl. } \\
(\mathrm{J} 2000)\end{array}$ & Date & $\begin{array}{l}\text { Exp. Time } \\
\text { (ks) }\end{array}$ & Filter & Notes \\
\hline$S 1$ & 0112660101 & $05: 35: 25.98$ & $-05: 54: 35.6$ & 2001 Sep 15 & 22.5 & Thick & Archive- $\_$Ori \\
\hline$S 2$ & 0503560701 & $05: 34: 49.90$ & $-06: 21: 53.0$ & 2007 Sep 10 & 55.4 & Medium & This program \\
\hline$S 3$ & 0089940301 & $05: 36: 22.30$ & $-06: 22: 19.0$ & 2001 Mar 18 & 45.8 & Thin 1 & Archive_-V380 Ori \\
\hline$S 4$ & 0503560601 & $05: 37: 30.20$ & $-06: 42: 03.9$ & 2007 Sep 08 & 55.1 & Medium & This program \\
\hline$S 5 a$ & 0205150401 & $05: 38: 18.10$ & $-07: 02: 24.9$ & 2004 Mar 27 & 4.6 & Medium & Archive_-V883 Ori \\
\hline$S 5 b$ & 0205150501 & $05: 38: 18.10$ & $-07: 02: 24.9$ & 2004 Aug 25 & 7.7 & Medium & Archive_-V883 Ori \\
\hline$S 6$ & 0503560101 & $05: 39: 35.10$ & $-07: 21: 09.3$ & 2007 Aug 27 & 48.3 & Medium & This program \\
\hline$S 7$ & 0503560401 & $05: 40: 33.30$ & $-07: 39: 07.7$ & 2007 Aug 31 & 53.0 & Medium & This program \\
\hline$S 8$ & 0503560201 & $05: 41: 41.37$ & $-07: 57: 19.1$ & 2007 Sep 20 & 60.0 & Medium & This program \\
\hline$S 9$ & 0503560301 & $05: 42: 54.00$ & $-08: 15: 52.6$ & 2007 Sep 20 & 41.6 & Medium & This program \\
\hline$S 10$ & 0503560501 & $05: 42: 52.70$ & $-08: 34: 37.7$ & 2008 Feb 23 & 46.5 & Medium & This program \\
\hline \multicolumn{8}{|c|}{ Northern Fields } \\
\hline Field Name & ObsID & $\begin{array}{c}\text { R.A. } \\
\text { (J2000) }\end{array}$ & $\begin{array}{c}\text { Decl. } \\
(\mathrm{J} 2000)\end{array}$ & Date & $\begin{array}{c}\text { Exposure Time } \\
(\mathrm{ks})\end{array}$ & Filter & Notes \\
\hline$N 1$ & 0049560301 & $05: 35: 10.81$ & $-04: 34: 06.8$ & 2002 Sep 15 & 26.3 & Thick & Archive \\
\hline$N 2 a$ & 0093000101 & $05: 35: 27.70$ & $-05: 05: 56.5$ & 2001 Mar 25 & 60.0 & Medium & Archive \\
\hline$N 2 b$ & 0093000301 & $05: 35: 27.70$ & $-05: 05: 56.5$ & 2001 Mar 26 & 17.3 & Medium & Archive \\
\hline$N 2 c$ & 0134531601 & $05: 35: 27.70$ & $-05: 05: 56.5$ & 2003 Mar 15 & 20.7 & Medium & Archive \\
\hline$N 2 d$ & 0134531701 & $05: 35: 27.70$ & $-05: 05: 56.5$ & 2003 Sep 15 & 20.9 & Medium & Archive \\
\hline$N 3 a$ & 0212480301 & $05: 34: 44.70$ & $-05: 33: 41.3$ & 2005 Feb 18 & 19.9 & Medium & Archive-V1118 Ori \\
\hline$N 3 b$ & 0212480401 & $05: 34: 44.70$ & $-05: 33: 41.3$ & 2005 Mar 21 & 19.8 & Medium & Archive_-V1118 Ori \\
\hline$N 3 c$ & 0212481101 & $05: 34: 44.70$ & $-05: 33: 41.3$ & 2005 Sep 08 & 12.4 & Medium & Archive_-V1118 Ori \\
\hline
\end{tabular}

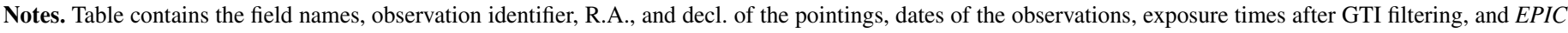
filters used during exposures. For ObsID 0212481101 only the PN exposure has been analyzed.

Table 1 gives a $\log$ of the observations. The SOXS fields in L1641 are labeled $S 1-S 10$. Typical exposure times are of order 50-60 ks for the seven SOXS fields and the $\iota$ Orionis field, while exposures are shorter $(\sim 15-20 \mathrm{ks})$ for the V380 Ori and V883 Ori fields. The field of V883 Ori has been observed twice, with observations taken five months apart. These two archival observations are called $S 5 a$ and $S 5 b$ and are analyzed together to improve the count statistics (ObsIDs 0205150401 and 0205150501). The Medium filter was used to screen UV contamination for all fields except for $\iota$ Orionis and V380 Ori fields, where Thick and Thin1 filters were used, respectively. The differences in the effective area among these filters are small above $0.7-0.8 \mathrm{keV}$, where most of the X-rays from YSOs are detected. We have also considered other archival fields containing the ONC (named $N 1, N 2, N 3$, Figure 2) to derive $\mathrm{X}$-ray luminosity distributions and for comparison with L1641.

The point-spread function (PSF) of XMM-Newton has a core width of about $5^{\prime \prime}$. In contrast to Chandra, it remains quite constant at increasing off-axis angles. Its shape becomes less circular and more elongated at high off-axis angles. At outer regions of the field of view the sensitivity is mainly affected by vignetting, and only at very high off-axis angles by the change of shape of the PSF. In our analysis, this problem affects the region between fields $N 2$ and $N 3$ which contains the core of the ONC. This region has been excluded from subsequent analysis, whereas in the southern fields $S 1-S 10$ source confusion is not an issue.

\subsection{XMM-Newton Data Analysis}

The initial screening and analysis of the data were performed using $S A S$ software ver. 8.0. From the Observation Data Files, we obtained fits tables of events recorded by the EPIC cameras PN, MOS1, and MOS2 with calibrated energies and astrometry.
Subsequently, we limited events to those within the $0.3-8.0 \mathrm{keV}$ band, removed the events out of the field of view, and selected only single and double events (PATTERN $\leqslant 12$ ). We have also screened the exposure times (good time intervals, GTIs) of each field to improve the signal-to-noise ratio $(\mathrm{S} / \mathrm{N})$ of faint sources in order to effectively detect them. This step is necessary to remove the intervals of high background due to, for example, solar activity. We used the algorithm of Sciortino et al. (2001) as elaborated by Damiani et al. (2003) which maximizes the $\mathrm{S} / \mathrm{N}$ of the whole image, by assuming that the variability in the global light curve is determined by the background variability only. We choose the GTIs that realize the maximum $\mathrm{S} / \mathrm{N}$ and this defines a limiting threshold in the count rate.

We performed source detection with a wavelet-based code derived from an analogous code developed for the ROSAT and Chandra instruments (Pwxdetect; see Damiani et al. 1997; Sciortino et al. 2001). The version of the code is adapted to detect sources in a list of XMM-Newton EPIC images combining the original unbinned event files in the weighted sum of MOS and PN images, and with the multi-scale approach inherited from its ROSAT predecessor (see also Pillitteri et al. 2010).

In order to choose a proper threshold for detection, several iterations of source detection on background-only images have been performed. We examined the cumulative distribution of the significance of detected spurious sources in these images and have chosen a threshold value to limit false detections to 1-2 per field. The threshold for significance of source detection was $\geqslant 4.5 \sigma$ of the local background mean in most cases. In the short exposure fields, the threshold has been chosen slightly lower, following the results of the simulations. The observations of the field of V883 Ori (ObsIDs 0205150401 and 0205150501) were merged into a single observation prior to analysis, as they are identical pointings. The final list of X-ray sources has been 


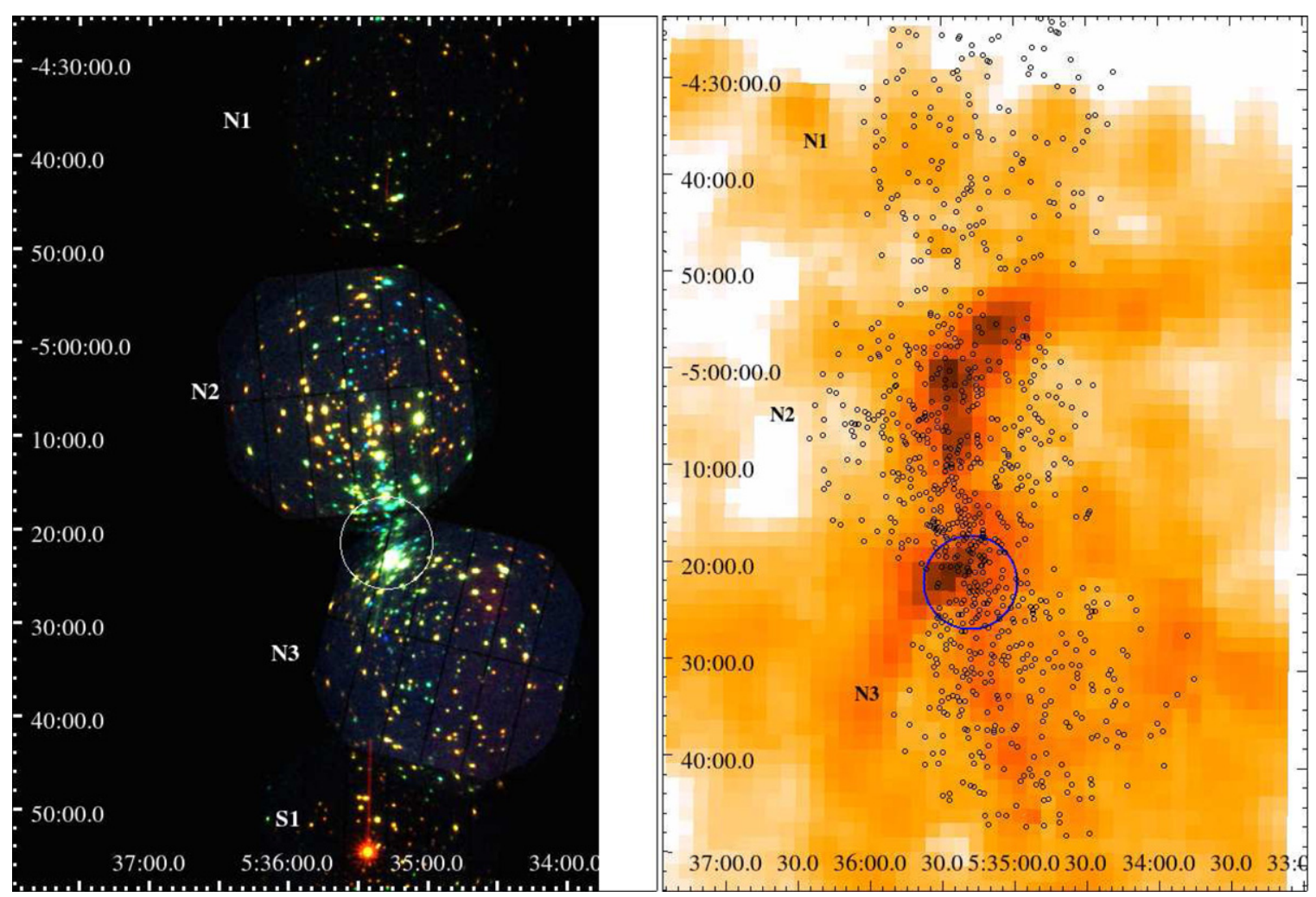

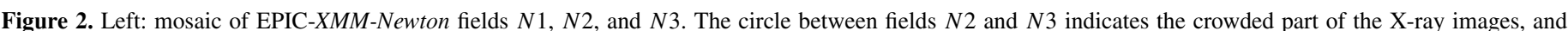

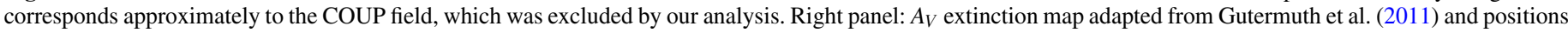

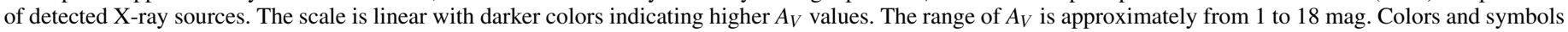
are same as in Figure 1.

(A color version of this figure is available in the online journal.)

checked to remove false sources that are caused by bright pixels or spikes around very bright sources. Table 2 reports 1060 sources detected in fields $S 1-S 10$.

We defined extraction regions for sources and background to select events and produce light curves and spectra by means of the SAS task regions. The task defines circular regions for each source and tries to maximize the $\mathrm{S} / \mathrm{N}$ with respect to a local background while avoiding overlap between nearby sources. Events, light curves, and spectra have been extracted for each source. Spectra were obtained with the SAS task especget. For each source, the task creates the source and background spectra, calculates the ancillary response file, and writes the area scaling factors for background and source spectra into the respective headers of the files. For PN spectra, it associates and writes in the spectrum file the correct response matrix (RMF) from the list of pre-calculated response files in the $X M M-N e w t o n$ archive, while for MOS, due to the peculiar form of the RMF and the different contributions to the RMF, ad hoc RMFs have been calculated for each source by using the task rmfgen. For sources with more than 500 total counts (232 out of 1060) as determined from the wavelet analysis, spectra from MOS1, MOS2, and PN were grouped to have at least 25 counts bin $^{-1}$ and fit simultaneously with XSPEC software ver. 12.7 (Arnaud et al. 1999) using one or two thermal APEC models (Smith et al. 1999) with foreground absorption and with both fixed and varying metallicity $(Z)$. We have verified that reliable spectral fits require approximately a minimum of 500 total (MOS1+MOS2+PN) counts. We simulated spectra with different count statistics and different shape to assess the validity of the 500 count threshold. Fainter sources are not suitable for meaningful spectral analysis because uncertainties become large and it is hard to distinguish between a thermal and a non-thermal (e.g., a power law) spectrum.

As a general criterion to determine the parameters from the best-fit modeling, we used the $\chi^{2}$ statistics, accepting as a good fit the models with the lowest number of free parameters that realize $P\left(\chi^{2}>\chi_{0}^{2}\right)>0.01$. In the case of two very bright sources, we accepted the best-fit parameters from the 2-T and $\mathrm{Z}$ variable model even if $P\left(\chi^{2}>\chi_{0}^{2}\right)<0.01$ because the overall shape of the spectrum was well fit. Only near a few blends of lines was the agreement with the best-fit model poor.

In one case (SOXS 258), we adopted a more specialized model spectrum constructed from a sum of one VAPEC + one APEC model, plus absorption. The Fe and $\mathrm{Z}$ abundance were linked together: the VAPEC component takes into account a cool component while the APEC component models the high-energy part of the spectrum. Specific abundances were $[\mathrm{Fe} / \mathrm{H}]=0.13$ and $[\mathrm{O} / \mathrm{H}]=0.34$, while temperatures were found to be $k T_{1}=0.2 \mathrm{keV}, k T_{2}=0.5 \mathrm{keV}$, and absorption $N_{\mathrm{H}}=1.5 \times 10^{20} \mathrm{~cm}^{-2}$. The results of the best-fit procedure for the 232 bright sources are listed in Table 3. The rateto-flux conversion factors ( $\mathrm{CFs}$ ) for faint sources have been calculated by taking the median of temperatures and absorption obtained from the spectral fits of the bright sources for each IR class. With PIMMS software and by assuming an absorbed 1-T APEC thermal model, the CFs have been calculated for the MOS camera and the filter used given that we derived the rates for MOS with the wavelet detection code. The $\mathrm{CF}$ values are: $1.5 \times 10^{-11}, 9.74 \times 10^{-12}$, and $6.71 \times 10^{-12} \mathrm{erg} \mathrm{cm}^{-2}$ count $^{-1}$ for Class I-III objects, respectively. 
Table 2

List of X-Ray-Detected Sources

\begin{tabular}{|c|c|c|c|c|c|c|c|}
\hline Id. & $\begin{array}{c}\text { R.A. (J2000) } \\
\text { (deg) }\end{array}$ & $\begin{array}{c}\text { Decl. (J2000) } \\
\text { (deg) }\end{array}$ & $\begin{array}{c}\text { Off-axis } \\
\left(^{\prime}\right)\end{array}$ & Counts & $\begin{array}{c}\text { Rate } \\
\left(\text { count ks }{ }^{-1}\right)\end{array}$ & $\begin{array}{c}\text { Exp. Time } \\
\text { (ks) }\end{array}$ & ObsID \\
\hline 1 & $05: 33: 49.5$ & $-06: 18: 05.9$ & 14.14 & $33 \pm 9$ & $0.74 \pm 0.2$ & 27.67 & 0503560701 \\
\hline 2 & $05: 33: 50.3$ & $-06: 21: 31.6$ & 13.79 & $625 \pm 38$ & $13.69 \pm 0.83$ & 27.94 & 0503560701 \\
\hline 3 & $05: 33: 53.3$ & $-06: 17: 11.1$ & 13.40 & $376 \pm 30$ & $8.15 \pm 0.65$ & 28.25 & 0503560701 \\
\hline 4 & $05: 33: 58.5$ & $-06: 15: 14.2$ & 12.84 & $96 \pm 17$ & $1.73 \pm 0.3$ & 34.08 & 0503560701 \\
\hline 5 & 05:34:00.0 & $-06: 31: 14.2$ & 15.60 & $202 \pm 23$ & $4.51 \pm 0.51$ & 27.37 & 0503560701 \\
\hline 6 & $05: 34: 02.1$ & $-06: 27: 07.3$ & 12.66 & $125 \pm 20$ & $1.49 \pm 0.24$ & 51.16 & 0503560701 \\
\hline 7 & 05:34:03.9 & $-06: 16: 03.7$ & 11.28 & $396 \pm 46$ & $10.14 \pm 1.2$ & 23.91 & 0503560701 \\
\hline 8 & 05:34:04.0 & $-06: 13: 30.2$ & 12.49 & $71 \pm 15$ & $0.86 \pm 0.19$ & 50.69 & 0503560701 \\
\hline 9 & $05: 34: 06.3$ & $-06: 30: 46.5$ & 14.16 & $778 \pm 43$ & $14.12 \pm 0.78$ & 33.72 & 0503560701 \\
\hline 10 & $05: 34: 06.6$ & $-06: 24: 13$ & 10.37 & $629 \pm 48$ & $11.88 \pm 0.9$ & 32.37 & 0503560701 \\
\hline
\end{tabular}

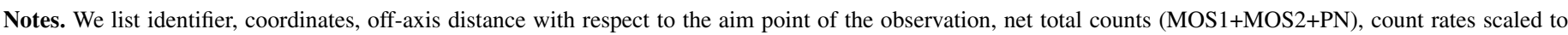
MOS sensitivity, sum of exposure times from EPIC cameras, and XMM observation identifier. Counts and count rates are given in the 0.3-8.0 keV band.

(This table is available in its entirety in a machine-readable form in the online journal. A portion is shown here for guidance regarding its form and content.)

Table 3

Best-fit Parameters $1 \sigma$ Errors Obtained from the Modeling of X-Ray Spectra

\begin{tabular}{|c|c|c|c|c|c|c|c|c|c|c|}
\hline SOXS Id. & Model & $\chi_{\text {red }}^{2}$ & dof & $\begin{array}{l}N_{\mathrm{H}} / 10^{20} \\
\left(\mathrm{~cm}^{-2}\right)\end{array}$ & $\begin{array}{c}k T 1 \\
(\mathrm{keV})\end{array}$ & $\begin{array}{c}10^{5} \times \text { norm }_{1} \\
\left(\mathrm{~cm}^{-5}\right)\end{array}$ & $\begin{array}{c}k T 2 \\
(\mathrm{keV})\end{array}$ & $\begin{array}{c}10^{5} \times \text { norm }_{2} \\
\left(\mathrm{~cm}^{-5}\right)\end{array}$ & $Z$ & $\begin{array}{c}10^{14} \times f_{\mathrm{X}} \\
\left(\mathrm{erg} \mathrm{s}^{-1} \mathrm{~cm}^{-2}\right)\end{array}$ \\
\hline 4 & $2 \mathrm{t} \_z v a r$ & 1.08 & 329 & $14.21 \pm 0.73$ & $10.86 \pm 7.65$ & $53.37 \pm 8.78$ & $1.01 \pm 0.03$ & $283.8 \pm 39.64$ & 0 & 219.73 \\
\hline 6 & 1t_zvar & 1.16 & 8 & $1.94 \pm 5.04$ & $0.86 \pm 0.09$ & $9.58 \pm 6.17$ & $\ldots$ & $\ldots$ & $\ldots$ & 5.86 \\
\hline 10 & 1t_zvar & 1.44 & 38 & $8.17 \pm 2.28$ & $0.76 \pm 0.07$ & $24.53 \pm 9.61$ & $\ldots$ & $\ldots$ & $\ldots$ & 11.31 \\
\hline 11 & $1 \mathrm{t}$ & 0.95 & 44 & $96.54 \pm 22.34$ & $17.13 \pm 13.5$ & $9.83 \pm 1.61$ & $\ldots$ & $\ldots$ & $\ldots$ & 16.12 \\
\hline 16 & 1t_zvar & 1.45 & 34 & $17.35 \pm 3.93$ & $0.95 \pm 0.13$ & $19.62 \pm 10.63$ & $\ldots$ & $\ldots$ & $\ldots$ & 11.51 \\
\hline 18 & $2 \mathrm{t} \_z v a r$ & 1.65 & 21 & $10.88 \pm 8.1$ & $0.75 \pm 0.12$ & $5.8 \pm 5.08$ & $0.22 \pm 0.06$ & $10.6 \pm 20.88$ & 0.1 & 3.28 \\
\hline 20 & 1t_zvar & 1.01 & 110 & $4.12 \pm 1.13$ & $0.95 \pm 0.04$ & $24.09 \pm 4.56$ & $\ldots$ & $\ldots$ & $\ldots$ & 14.46 \\
\hline 27 & $1 \mathrm{t}$ & 1.56 & 39 & 0 & $1.54 \pm 0.09$ & $3.99 \pm 0.37$ & $\ldots$ & $\ldots$ & $\ldots$ & 3.96 \\
\hline 32 & 1t_zvar & 1.24 & 33 & $2.51 \pm 2.2$ & $0.92 \pm 0.07$ & $10.37 \pm 3.83$ & $\ldots$ & $\ldots$ & $\ldots$ & 6.32 \\
\hline 33 & $1 \mathrm{t}$ & 1.52 & 34 & 0 & $0.94 \pm 0.05$ & $1.41 \pm 0.19$ & $\ldots$ & $\ldots$ & $\ldots$ & 1.49 \\
\hline
\end{tabular}

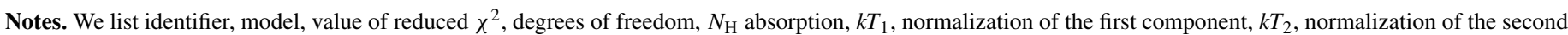
component, global abundance $Z$, and unabsorbed fluxes in $0.3-8 \mathrm{keV}$ band.

(This table is available in its entirety in a machine-readable form in the online journal. A portion is shown here for guidance regarding its form and content.)

For the northern fields $N 1, N 2$, and $N 3$, we have followed the same procedure of filtering and source detection as used for the southern fields. The total number of detected sources in fields $N 1, N 2$, and $N 3$ is 864 . From source count rates and rate-to-flux CFs derived for the L1641 sources, we have derived fluxes and luminosities in the $0.3-8.0 \mathrm{keV}$ band.

\subsection{Spitzer Data}

The analysis of the Spitzer data and the IR catalog are discussed in Megeath et al. (2012). We used the IR catalog of Megeath et al. of objects detected in the four Infrared Array Camera (IRAC) bands and MIPS [24] band. The PSF width of Spitzer IRAC is $\sim 1^{\prime \prime} 6$ and thus better than the $X M M$-Newton PSF, while the PSF width of MIPS [24] is slightly worse $\left(d \sim 6^{\prime \prime}\right)$. The XMM-Newton fields are almost fully contained in the Spitzer sky coverage, with the exception of small portions of fields $S 2, S 3, S 8$, and $S 9$, where a total of 16 X-ray sources lie outside the field of view of Spitzer.

The stellar objects in the catalog have been separated into infrared "Classes" (see, e.g., Lada \& Adams 1992; Allen et al. 2004; Megeath et al. 2004) following a prescription similar to that used by Gutermuth et al. (2008, 2009), Kryukova et al. (2012), and Megeath et al. (2012). The classification is based on cuts in a multidimensional color space, including $J, H, K_{S}$, all four IRAC channels, and the MIPS $24 \mu \mathrm{m}$ band. Examples of cuts in the multidimensional color space are shown in Figure 3. Color-magnitude diagrams, especially [4.5] versus [4.5] - [8.0] (left top panel in Figure 3) help to identify the type of objects more reliably since active galactic nuclei (AGNs), while red, tend to be fainter than YSOs. Sources are classified as either PMS stars with disks or protostars based primarily on the slope of their spectral energy distribution (SED), as described in Kryukova et al. (2012) and Megeath et al. (2012). Red objects fainter than [4.5] = $14 \mathrm{mag}$ are usually found to be galaxies or background stars. In fact, a histogram of [4.5] mag (Figure 3) shows a double-peaked distribution with a minimum between the two peaks at $[4.5]=14$ mag. The main peak is composed of objects likely belonging entirely to the cloud; the second peak is formed by distant objects, including background stars and galaxies.

In the XMM-Newton fields $S 1-S 10$ there are 650 Class II objects (called stars with disks in Megeath et al. 2012) and 147 Class 0-I and flat-spectrum objects (protostars in Megeath et al. 2012). The total Spitzer survey of Orion A and B clouds is comprised of 3480 YSOs, 2992 Class II objects, and 428 protostars. To these should be added another 50 protostar candidates and 10 sources detected only in Spitzer-MIPS which are likely protostars (Megeath et al. 2012). X-rays can effectively inform us about the Class III/WTTS population of L1641. We have identified Class III candidates as those objects with 

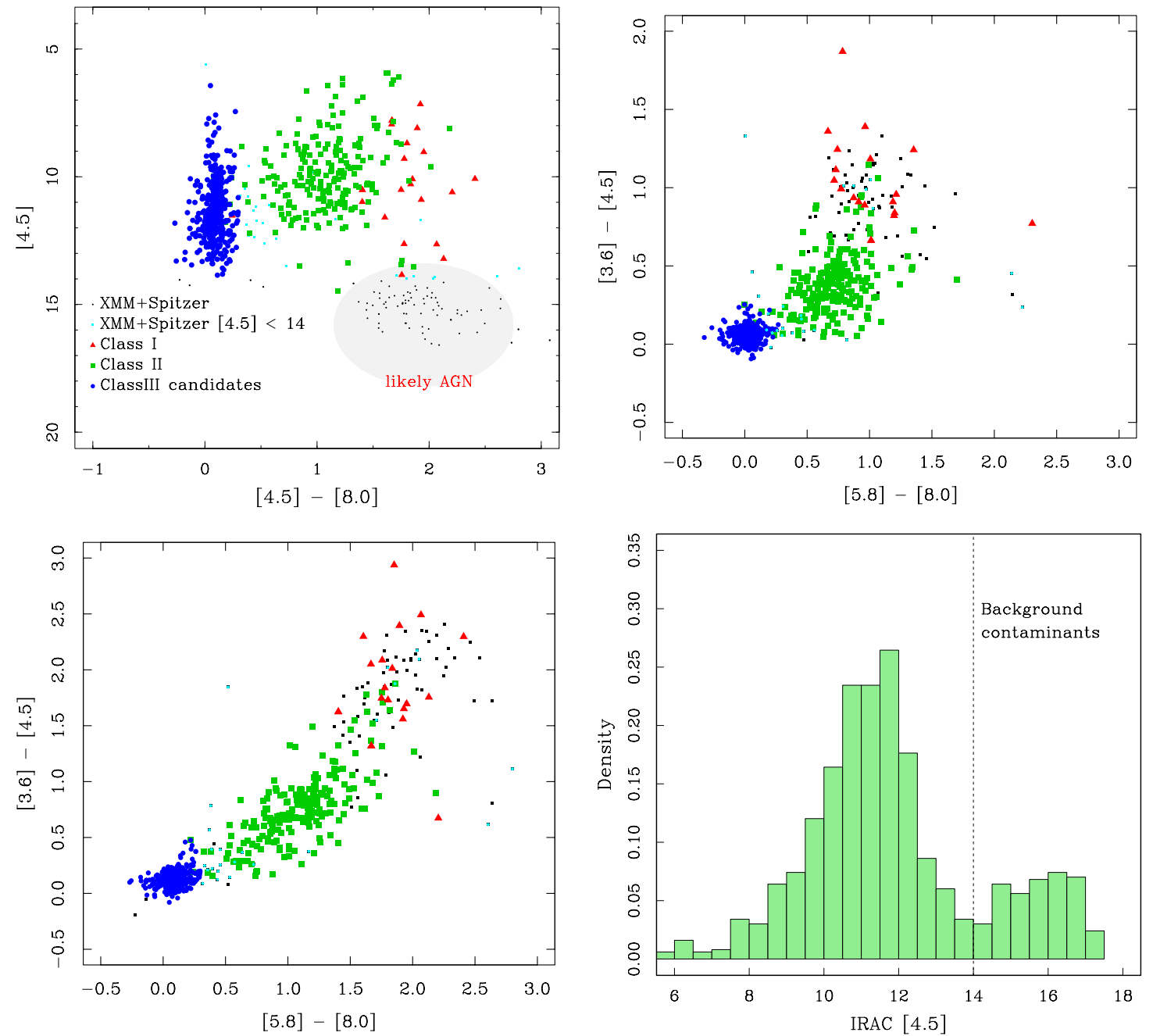

Figure 3. IRAC color-color and color-magnitude diagrams of SOXS objects. Triangles are protostars, squares are Class II stars, as derived from Spitzer data alone. Circles are Class III candidates. The histogram of IRAC [4.5] mag shows a bi-modal population. The objects in the fainter peak with IRAC [4.5] > 14 mag are likely background objects.

(A color version of this figure is available in the online journal.)

$\mathrm{X}$-ray detection, and IRAC [4.5] $\leqslant 14 \mathrm{mag}$ and IRAC color $[4.5]-[8.0] \leqslant 0.5$ mag (see Figure 3).

Table 4 lists the matches between X-ray and IR sources.

\section{RESULTS}

Figures 1 and 2 (left panels) show a red-green-blue (RGB) image of the entire survey obtained with EPIC XMM-Newton. We have labeled the fields as in Table 1. Sources that are red to yellow have softer, less absorbed spectra than green or blue sources. The right panels in both figures show the $A_{V}$ extinction map, adapted from Figure 4 of Gutermuth et al. (2011), and the X-ray sources detected in XMM-Newton images. The X-ray sources show some degree of clustering where the extinction is higher, for example, in fields $S 5, S 6$, and $S 10$. We detected a total of 1060 X-ray sources in the 10 southern SOXS fields $(S 1-S 10)$ with more than $4.5 \sigma$ significance. The 1060 sources include 31 sources detected in the overlapping regions between two adjacent pointings. We matched the X-ray positions with the positions of near- and mid-IR sources from the Spitzer catalog, using an offset limit of $5^{\prime \prime}$, and iterated the cross identification process three times to remove systematic offsets along R.A. and decl. of the X-ray sources. The final offsets for sources with off-axis less than $10^{\prime}$ from the aim point have a median of $1^{\prime \prime} .4$, and a $10 \%-90 \%$ range of $0.7-33^{\prime \prime} 3$. For sources at off-axis $>10^{\prime}$, the values are similar: the median of offsets is $1^{\prime \prime} .7$, and $0.7-33^{\prime \prime} \cdot 7$ is the $10 \%-90 \%$ range.

We find that 972 X-ray sources have at least a match with an object in the 2MASS+Spitzer catalog. There are 69 cases of $\mathrm{X}$-ray sources with multiple counterparts among the IR objects: 66 cases of matches with two IR objects and 3 cases of triple matches.

We have also considered X-ray sources with a significance of detection lower than the $4.5 \sigma$ threshold in order to identify other faint X-ray emission among known Class I and Class II objects. With this choice, we find 9 protostars and 19 Class II objects with X-ray detection, which have a significance between $3 \sigma$ and $4.5 \sigma$ of the local background. On average, one additional Class I and two Class II objects per field are found, respectively. The probability of a chance match of these stars is of order of $10^{-3}$. However, in the following analysis we will not consider these additional $\mathrm{X}$-ray detections and we will only discuss the sample of 1060 sources detected with significance above $4.5 \sigma$. In particular, we cannot extend the list of Class III candidates by considering low significance detections because of the high number of objects in the IR catalog that appear as normal photospheres and, consequently, the high likelihood of matching spurious sources. Table 2 reports the number of Spitzer 
Table 4

List of X-Ray and IR Matches

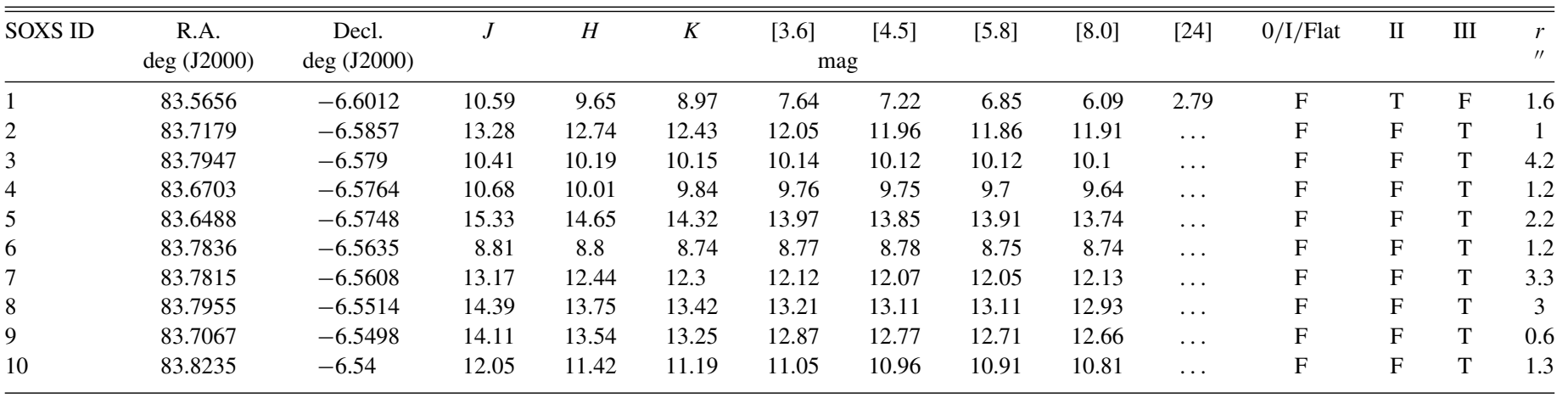

Notes. Columns are: SOXS source number, IR coordinates, 2MASS $J, H, K$ magnitudes, IRAC [3.6], [4.5], [5.8], [8.0], MIPS [24] band magnitudes, a flag indicating Class 0/I/flat-spectrum object, Class II object, or Class III candidate status, and the match radius between X-ray and IR positions. X-ray sources with more than a potential IR counterpart have multiple entries.

(This table is available in its entirety in a machine-readable form in the online journal. A portion is shown here for guidance regarding its form and content.)

Table 5

List of Candidates with MIPS [24] Excesses, Indicating That These May Have Transitional Disks, and Two Additional Class III Candidates That are IR Objects with Incomplete Spitzer Photometry and a Match with X-Ray Sources

\begin{tabular}{|c|c|c|c|c|c|c|c|c|c|c|c|}
\hline Id & $\begin{array}{c}\text { R.A. } \\
\text { (J2000, deg) }\end{array}$ & $\begin{array}{c}\text { Decl. } \\
(\mathrm{J} 2000, \text { deg })\end{array}$ & $J$ & $H$ & $K_{s}$ & $\begin{array}{l}{[3.6]} \\
(\mathrm{mag})\end{array}$ & [4.5] & {$[5.8]$} & {$[8.0]$} & [24] & Notes \\
\hline 13 & 83.525907 & -6.5125008 & 11.59 & 10.84 & 10.59 & 10.42 & 10.39 & 10.34 & 10.30 & 5.68 & $\mathrm{a}, \mathrm{b}$ \\
\hline 33 & 83.905423 & -6.3907431 & 12.52 & 11.87 & 11.61 & 11.40 & 11.32 & 11.27 & 11.19 & 5.94 & $\mathrm{a}$ \\
\hline 136 & 84.385151 & -6.6514246 & 12.97 & 12.28 & 12.07 & 11.83 & 11.77 & 11.71 & 11.59 & 6.88 & $\mathrm{a}$ \\
\hline 163 & 85.734935 & -8.6293451 & 13.74 & 12.87 & 12.57 & 12.33 & 12.27 & 12.19 & 12.12 & 9.14 & $\mathrm{c}$ \\
\hline 173 & 85.269234 & -7.7611424 & 11.63 & 10.67 & 10.30 & 10.11 & 10.04 & 9.916 & 9.80 & 6.17 & a \\
\hline 269 & 85.475248 & -7.8314385 & 12.23 & 11.45 & 11.08 & 10.80 & 10.72 & 10.67 & 10.59 & 6.52 & a \\
\hline 276 & 84.907365 & -7.4397500 & 16.23 & 13.50 & 12.12 & 11.34 & 11.08 & 10.86 & 10.86 & 7.48 & a \\
\hline 281 & 84.640908 & -7.2841951 & 11.09 & 10.36 & 10.14 & 10.01 & 9.99 & 9.96 & 9.91 & 7.34 & a \\
\hline 383 & 83.862722 & -5.8145652 & 10.48 & 9.99 & 9.80 & 9.035 & 8.74 & 8.37 & 7.73 & 4.68 & a \\
\hline 468 & 83.946119 & -6.1958249 & 13.86 & 13.25 & 12.97 & 12.59 & 12.48 & 12.39 & 12.36 & 8.86 & d \\
\hline 61 & 83.820037 & -6.3040907 & $\ldots$ & $\ldots$ & $\ldots$ & $\ldots$ & $\ldots$ & 11.05 & $\ldots$ & $\ldots$ & e \\
\hline 126 & 84.510766 & -6.7102342 & $\ldots$ & $\ldots$ & $\ldots$ & 14.77 & 14.68 & $\ldots$ & $\ldots$ & $\ldots$ & $\mathrm{f}$ \\
\hline
\end{tabular}

Notes.

a Strong, isolated detection.

${ }^{\mathrm{b}}$ Off of the main cloud region.

${ }^{\mathrm{c}}$ Faint, near airy ring.

${ }^{\mathrm{d}}$ Faint, in nebulosity, near the edge of detection; may be elongated; could be a contamination.

e Double source.

${ }^{\mathrm{f}}$ Faint, only detected in IRAC [3.6] and [4.5].

objects in fields $S 1-S 10$, the number of X-ray sources, and the rate of detections from the list of $1060 \mathrm{X}$-ray sources. We also give the ratios of Class I to Class II objects and of Class III to Class II objects. Analogous numbers for the fields $N 1-N 3$ are biased and incomplete because of source confusion in X-ray and IR bands around the core of the ONC. By following the criteria in Section 2.2, we find 489 Class III candidates. Among these 489 objects, 10 have strong MIPS $24 \mu \mathrm{m}$ band excesses that suggest they are transitional disk objects, i.e., young stars with a depleted inner circumstellar disk. Two further objects have incomplete photometry in IRAC and MIPS bands that prevents the detection of IR excesses. One object is a very faint IR source with detection only in the [3.6] and [4.5] bands; the other object is actually a double source with confused photometry. The positions and the IR photometry of the transitional disk objects and the two faint objects are shown in Table 5.

We give an estimate of the number of extragalactic sources that we expect to find by using PIMMS and the limiting count rate given in Section 3.1. To estimate a limiting flux for these objects, we assumed a spectrum due to an absorbed power-law model with index 1.4 for the case of self-absorbed AGNs and 1.9 for AGNs without self-absorption. Then we derived the number of expected extragalactic sources per square degree by using the $\log N \log S$ model of the POMPA code (Gilli et al. 2007; see also Puccetti et al. 2009). The model predicts $\sim 70-90$ sources $\mathrm{deg}^{-2}$ in $0.5-10 \mathrm{keV}$ or about $120-150$ sources in SOXS. Figure 3 shows about 75 objects detected in X-rays and with IRAC colors and magnitudes consistent with those of background AGNs. This number is lower by a factor of $\sim 1.6-2$ with respect to the number of expected extragalactic sources. A possible bias of this estimate is the presence of the cloud of Orion A and its effect on X-ray fluxes.

Nineteen X-ray sources are unidentified in IR or optical catalogs. Statistically, about 10-15 spurious sources are expected for the adopted detection threshold, as estimated from the simulations of background-only images. We expect that the spurious 
sources are among those with the lowest significance. We thus have an excess of about 5 to 10 unidentified true sources. Given they are not matched in the IR catalog, their nature could be extragalactic or they are still YSOs embedded in bright nebulosity that hampers IR detection.

\subsection{Sensitivity of the Survey}

We have calculated sensitivity maps for each field based on the count rate threshold used for the source detection. All southern fields $(S 1-S 10)$, except those pointed toward V883 Ori $(S 5 a+b)$ and $\iota$ Ori $(S 1)$, have similar exposure times and similar instrumental sensitivity. Globally, the limiting sensitivity for a point source is $\sim 1.8 \times 10^{-4}$ count $\mathrm{s}^{-1}$ at the center and it is reduced to $\sim 10^{-3}$ count $\mathrm{s}^{-1}$ at $\sim 13^{\prime}$ off-axis. The combined V883 fields $(S 5 a+S 5 b)$ are shallower and have about half the sensitivity of other fields. The same is true for field $S 1$. Field $N 1$ is similar to $S 5 a+b$, while $N 2$ is about twice as deep as the rest.

To translate the rate sensitivity into limiting flux, we have considered a mono-thermal absorbed APEC model. The absorption column density is the main factor affecting the limiting flux, overwhelming the effects of a change in the plasma temperature. From spectral analysis, we have derived values of absorption as high as $N_{\mathrm{H}} \simeq 5 \times 10^{22} \mathrm{~cm}^{-2}$ with a median value of $N_{\mathrm{H}} \simeq 7 \times 10^{20} \mathrm{~cm}^{-2}$. Plasma temperature is found in the range between $k T=0.7 \mathrm{keV}$ and $k T=3 \mathrm{keV}$. We used the PIMMS software to estimate the limiting flux to the typical coronal emission of young stars. For a plasma with a temperature of $k T=1 \mathrm{keV}$ and for an absorbing gas column $N_{\mathrm{H}}$ ranging from $10^{19} \mathrm{~cm}^{-2}$ to $5 \times 10^{22} \mathrm{~cm}^{-2}$ (the range of values that we derived from the best-fit models), we have a limiting unabsorbed flux in the range of $F_{X}=1.4 \times 10^{-14}-6.6 \times 10^{-13} \mathrm{erg} \mathrm{s}^{-1} \mathrm{~cm}^{-2}$ $(0.3-8.0 \mathrm{keV})$, which is a variation of a factor of $\sim 47$. By considering a hotter plasma with $k T=3 \mathrm{keV}$, and varying $N_{\mathrm{H}}$ again from $10^{19} \mathrm{~cm}^{-2}$ to $5 \times 10^{22} \mathrm{~cm}^{-2}$, the limiting unabsorbed flux varies by a factor of four. The effect of absorption on the spectrum is less pronounced on the spectrum with a hotter kT because most of the emission is above $1 \mathrm{keV}$ where absorption is not relevant.

The limiting flux is determined mainly by the range of $N_{\mathrm{H}}$ absorption present in our survey rather than the effect due to a hotter or cooler plasma. Given the range of unabsorbed fluxes given above, at the distance of the ONC (414 pc; Menten et al. 2007), our survey can detect any source with an unabsorbed $X$-ray luminosity of $\log L_{X} \geqslant 28.5$ for a source placed on-axis. The EPIC camera has a quite stable PSF width within $\sim 10^{\prime}$. For sources placed off-axis by more than $10^{\prime}$, the decrease in sensitivity is about a factor of $\sim 2$.

\subsection{Completeness and Contamination}

The completeness of the IR catalog by Megeath et al. (2012) depends on the luminosities of the stars, the presence of a disk, and the brightness of the surrounding nebulosity. About $90 \%$ completeness is obtained for identifying YSOs at $K=13 \mathrm{mag}$. By using the tracks of Baraffe et al. (1998), this translates into $0.09 M_{\odot}$ at $1 \mathrm{Myr}$ and $0.175 M_{\odot}$ at $3 \mathrm{Myr}$, equivalent to a M4 type (Luhman 1999; Kenyon \& Hartmann 1995). In dense regions like the ONC the limit at the $K$ band can be $2-3$ mag brighter. In regions of low nebulosity like L1641 this effect is only limited to the densest parts and with an increase of the limiting magnitude of about 1 mag like in L1641 S across fields $S 8$ and $S 9$. As a consequence, the completeness of the survey in denser regions is decreased to $\sim 0.175 M_{\odot}$ for an age of $1 \mathrm{Myr}$ and $0.35 M_{\odot}$ at $3 \mathrm{Myr}$.

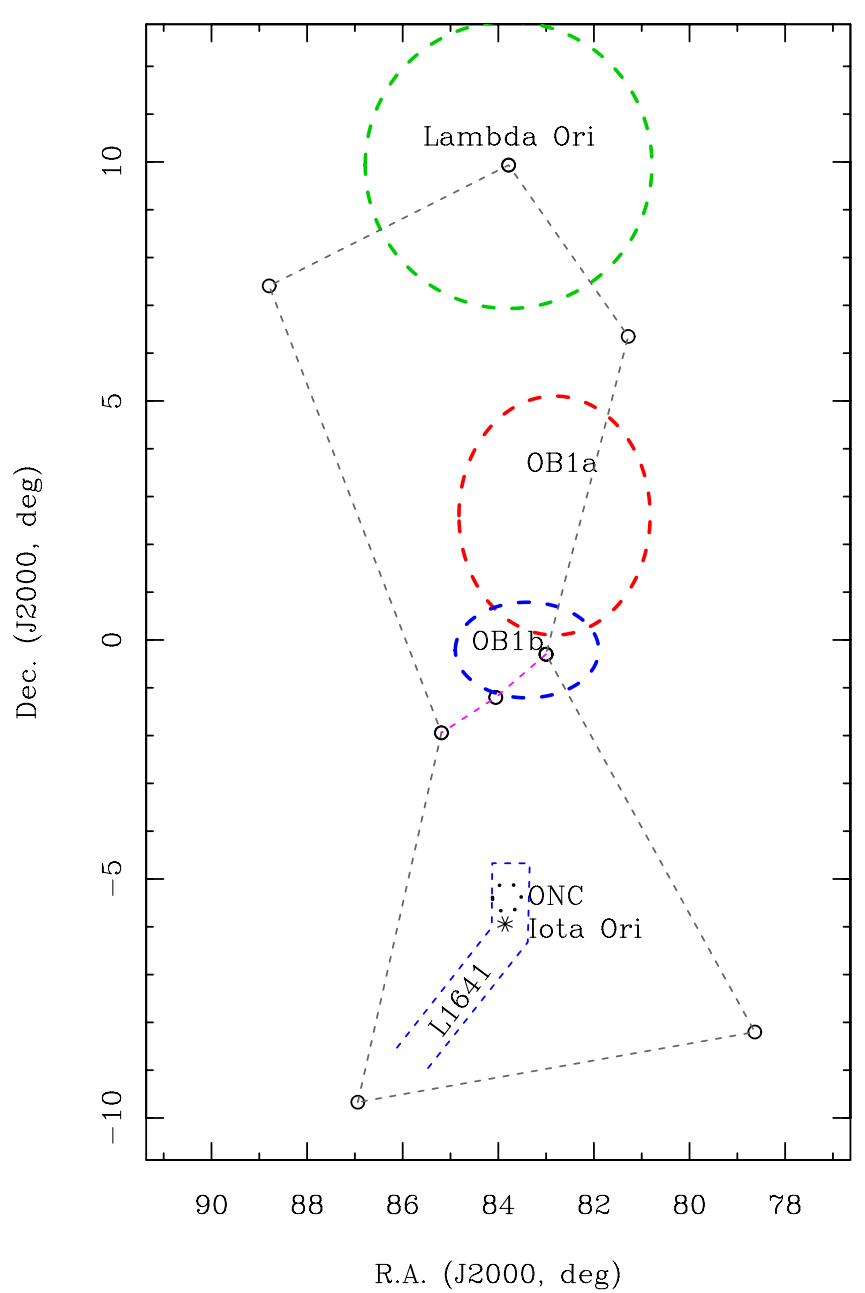

Figure 4. Geometry adopted for Monte Carlo simulations of contaminants from OB1a, OB1b, and the ONC. Locations of subgroups are taken from Bally (2008). The region around $\lambda$ Ori has been taken into account but given the distance and the age it gives a negligible contribution to the PMS population in L1641. The same is true for OB1b stars.

(A color version of this figure is available in the online journal.)

We assess the completeness of PMS stars detected in X-rays by comparing our results to the results from COUP (Feigelson et al. 2005; Getman et al. 2005), which constitutes the most complete sample obtained in X-rays for a star-forming region ( $~ 97 \%$ complete above spectral type M4). The XLD is modeled with a log-normal distribution centered at $\log L_{X}=29.3$ and standard deviation equal to 1 dex. If we assume that this XLD also yields T Tauri star (TTS) in other star formation regions (SFRs), we conclude that our X-ray observations have probed about $45 \%$ of the total population of TTs. Getman et al. (2006) have modeled the XLD of lightly obscured YSOs in COUP by means of a broken power law, with a knee at $\log L_{X}=2.5 \times 10^{30} \mathrm{erg} \mathrm{s}^{-1}$, while in other star-forming regions, like NGC 1333 and IC 348, a log-normal model still fits the data. By adopting the broken power-law model as in Getman et al. (2006), we obtain a completeness at a level of $46 \%$. The $\mathrm{X}$-ray sample is complete to $\log L_{X} \sim 29.5$, which corresponds approximately to the luminosity of an early K-type star at an age of 3 Myr.

The number of expected foreground contaminants in X-rays has been estimated by means of a log-normal distribution of the X-ray luminosities of field stars $\left(\mathrm{XLF}_{\text {field }}\right)$ with median $\log L_{X}=27.5$ and standard deviation $\sigma=1 \mathrm{dex}$ (see Favata 
Table 6

Expected Number of YSOs from Orion OB1a and the $\mathrm{ONC}$ in the Region of the Survey

\begin{tabular}{lcc}
\hline \hline Field & From OB1a & From the ONC \\
\hline$S 1$ & 2.6 & 104.9 \\
$S 2$ & 2.1 & 16.7 \\
$S 3$ & 2 & 13.7 \\
$S 4$ & 1.6 & 1 \\
$S 5$ & 1.3 & 0 \\
$S 6$ & 1.1 & 0 \\
$S 7$ & 0.9 & 0 \\
$S 8$ & 0.7 & 0 \\
$S 9$ & 0.5 & 0 \\
$S 10$ & 0.4 & 0 \\
\hline Total & 13.1 & 136.3 \\
\hline
\end{tabular}

\& Micela 2003 and references therein). We have assumed a conservative limiting flux of $f_{X, \lim }=1.5 \times 10^{-15} \mathrm{erg} \mathrm{s}^{-1} \mathrm{~cm}^{-2}$ and integrated all the contributions of the $\mathrm{XLD}_{\text {field }}$ up to the distance to Orion by assuming a density of field stars of $0.1 \mathrm{pc}^{-3}$. At most five foreground stars per field, $\simeq 50$ stars in all fields, are expected to be detected in SOXS.

We have also considered the possibility of detecting stars born in the northern part of Orion, namely, the Orion OB 1a and OB1b star-forming regions, that could have traveled south since their birth to the Orion A cloud and L1641. Figure 4 shows the location of the sites from which young stars could have migrated into L1641. To estimate the number of these objects, we considered a normal distribution of stellar velocities with zero mean and dispersion equal to $3 \mathrm{~km} \mathrm{~s}^{-1}$, and with an age of $10 \mathrm{Myr}, 3 \mathrm{Myr}$, and $1 \mathrm{Myr}$ for OB1a, OB1b, and the ONC, respectively (see Bally 2008; Buckle et al. 2012). We make the assumption that the group of stars from the northern regions is, on average, comoving with L1641. The stars with velocities of $3 \mathrm{~km} \mathrm{~s}^{-1}$ should have traveled on average distances of $30 \mathrm{pc}$, $9 \mathrm{pc}$, and $3 \mathrm{pc}$ from their birth place, which correspond to an angular distances of $5.32 \mathrm{deg}, 1.18 \mathrm{deg}$, and $0.42 \mathrm{deg}$, taking into account the distances of these groups (OB1a $330 \mathrm{pc}$, OB1b 440 pc (Sherry et al. 2004), and the ONC 414 pc). Furthermore, we have assumed a population of 1000 stars for OB1a, OB1b, and the ONC, respectively. We ran 10,000 Monte Carlo simulations of the stellar populations. The results are shown in Table 6 where we report the expected contaminants for each XMM-Newton field. The number of contaminants from OB1a decreases north to south with a total of $\sim 13 \pm 3$ stars. The contamination from $\mathrm{OB} 1 \mathrm{~b}$ and the $\lambda$ Ori ring is negligible due to the combined factors of distance and age, and thus their values are not reported in Table 6. About eight stars may have formed in OB1a and then traveled into NGC 1980 and L1641 N (fields $S 1+S 2+S 3+S 4$ ).

The contamination from the ONC is significant in the northern fields of SOXS. As shown in Table 6, the total number of objects traveling from the ONC to NGC 1980 and L1641 North is $\sim 136 \pm 12$ stars. Due to the difference in ages of the ONC and OB1c to which NGC 1980 belongs (1 Myr versus 5 Myr; Bally 2008), we expect that the population of YSOs coming from the ONC should be less evolved than the original population of stars in OB1c, and thus preferentially of earlier Classes (0/I, and II).

In summary, we estimate that less than 15 high-velocity stars from the subgroups north of Orion's Belt are present in SOXS fields. More pronounced is the number of stars that could have been born in the ONC and traveled into L1641 N and NGC 1980, and this number is about 130 YSOs. Contamination from the $\lambda$ Ori group and OB1b is negligible.

\section{THE POPULATION OF YOUNG STELLAR OBJECTS}

In this section we focus on the properties of the YSOs selected by means of their colors and the shape of their SEDs in Spitzer bands for Class I and Class II objects, and by their X-ray detection and absence of IR excess for Class III candidates. Table 7 summarizes the number of detections among Class I and Class II objects and Class III candidates.

The overall ratio of Class I to Class II objects detected by Spitzer is 0.23 (range: $0.20-0.25),{ }^{11}$ but from field to field this ratio varies between $0.08(0.05-0.11)$ and $0.64(0.47-0.86)$. In particular, the largest number of Class I YSOs with respect to Class II objects is found in the field S8 near L1641 South.

The ratio of Class III candidates and X-ray-detected Class II objects also varies across the fields. Values of the Class III to Class II ratio range from $0.87_{0.51}^{1.48}$ to $5.63_{4.14}^{8.01}$ with a mean ratio of 2.39(2.14-2.69). The number of Class III objects is always larger than the number of Class II objects except in the fields $S 5$ and S6. The highest ratio of Class III candidates with respect to Class II YSOs is observed in two distinct regions: the northern fields, $S 3$ and $S 1$, where the ratio ranges between three and five and in $S 9$, where it is more than five. This high ratio hints that a more evolved population of young stars is present toward $\iota$ Orionis and V380 Ori and in the subgroup belonging to L1641 $\mathrm{S}$ as we will discuss later.

A main goal of this survey is to estimate the number of young stars in L1641 North, L1641 South, and in the fields around V380, V883, and ` Ori. While we consider the sample of Class I and II YSOs detected and classified with Spitzer almost complete down to the $\sim$ M4 limit (Megeath et al. 2012), there is considerable uncertainty in the total PMS population of Class III stars. These appear as normal photospheres in the Spitzer bands, but they are characterized by high X-ray luminosity in a range from $5 \times 10^{28} \mathrm{erg} \mathrm{s}^{-1}$ to $10^{31} \mathrm{erg} \mathrm{s}^{-1}$. In Section 2.2, we have defined Class III candidates as the Spitzer counterparts of X-ray sources with IR magnitudes consistent with PMS stars $([4.5]<14 \mathrm{mag})$ at the distance of Orion A and without IR excess. The number of Class III candidates in fields $S 1-S 10$ is 489 . If we subtract 50 expected foreground stars, as estimated in Section 3.2, we find at least 439 Class III candidates. In Section 3.1, when discussing the sensitivity of the survey, we estimated a detection rate of X-ray sources of about $45 \%$. Taking this number as the detection rate of the Class III stars, we estimate a population of Class III stars of 1087 members in the case of 489 X-ray-detected stars, and 976 stars when correcting for the 50 foreground objects. To these numbers we add 650 to the Class II YSOs and 147 protostars present in the fields $S 1-S 10$, thus giving a total population of $\sim 1700-1884( \pm 90)$ YSOs, in all evolutionary stages, from protostars to diskless stars.

On the other hand, one could consider that the fraction of Class III stars detected in X-rays is the same as Class II objects $(\sim 30 \%)$. Under this hypothesis, the total sample of Class III stars should be about $1630 \pm 75$ or $\sim 1460 \pm 70$ when correcting for foreground objects. This leads to a total of $\sim 2260 \pm 110$ PMS stars above mid-M-type stars in the XMM-Newton fields $S 1-S 10$. One bias of this estimate is the implicit assumptions that Class II and III objects have similar luminosities and absorption, similar ages, and that all the young stars are at the

\footnotetext{
11 Obtained from the square root of numbers in Table 7.
} 
Table 7

Number of Spitzer Objects, X-Ray Detections, Class I and Class II YSOs in Each SOXS Field

\begin{tabular}{|c|c|c|c|c|c|c|c|c|c|c|c|}
\hline Field Name & $S 1$ & $S 2$ & $S 3$ & $S 4$ & $S 5 a+b$ & S6 & $S 7$ & $S 8$ & $S 9$ & $S 10$ & Total \\
\hline$N_{\text {Spitzer }}$ & 4232 & 4596 & 4641 & 4453 & 3341 & 4345 & 4259 & 4135 & 4259 & 4477 & $39891^{\mathrm{a}}$ \\
\hline Class I & 12 & 4 & 13 & 8 & 12 & 31 & 6 & 35 & 6 & 16 & 147 \\
\hline Class II & 69 & 36 & 110 & 39 & 147 & 54 & 62 & 55 & 62 & 43 & 650 \\
\hline $\mathrm{Cl} \mathrm{I} / \mathrm{Cl} \mathrm{II}$ & $17 \%$ & $11 \%$ & $12 \%$ & $21 \%$ & $8 \%$ & $57 \%$ & $10 \%$ & $64 \%$ & $10 \%$ & $37 \%$ & $23 \%$ \\
\hline$N_{X}$ & 202 & 149 & 161 & 104 & 83 & 69 & 53 & 84 & 107 & 48 & 1060 \\
\hline$N_{\mathrm{X}}$ in Spitzer & 200 & 156 & 158 & 104 & 76 & 66 & 51 & 78 & 96 & 56 & 1041 \\
\hline X det. Class I & 1 & 2 & 4 & 5 & 1 & 3 & 1 & 1 & 1 & 2 & 23 \\
\hline X det. Class II & 19 & 31 & 44 & 15 & 10 & 16 & 9 & 12 & 9 & 21 & 204 \\
\hline X det. Class III cand. & 107 & 113 & 78 & 15 & 50 & 14 & 23 & 20 & 49 & 27 & 489 \\
\hline Det. Fract. Cl I & 0.08 & 0.50 & 0.31 & 0.63 & 0.08 & 0.1 & 0.12 & 0.03 & 0.17 & 0.63 & $0.14^{b}$ \\
\hline Det. Fract. Cl II & 0.27 & 0.86 & 0.40 & 0.39 & 0.07 & 0.30 & 0.49 & 0.22 & 0.15 & 0.39 & $0.29^{\mathrm{b}}$ \\
\hline $\mathrm{X}$ det. $\mathrm{Cl} \mathrm{I} / \mathrm{Cl} \mathrm{II}$ & 0.09 & 0.33 & 0.06 & 0.10 & 0.05 & 0.19 & 0.11 & 0.08 & 0.11 & 0.10 & 0.11 \\
\hline $\mathrm{X}$ det. $\mathrm{Cl} \mathrm{III} / \mathrm{Cl} \mathrm{II}$ & 5.60 & 3.65 & 1.77 & 1.00 & 5.00 & 0.87 & 1.28 & 1.67 & 5.44 & 1.00 & $2.28^{b}$ \\
\hline
\end{tabular}

Notes.

a Some of the Spitzer objects are in overlapping fields, so that the sum of the numbers in each field is greater than the total of Spitzer objects present in all fields.

$\mathrm{b}$ This is the average detection rate, i.e., the sum of detections divided by the sum of objects in each field.

same distance. This is not exactly true for the region around $\iota$ Ori, where we find evidence of a closer and more evolved population that is depleted of protostars and Class II objects.

In summary, the total population of PMS stars and protostars in the portions of L1641 and toward ८ Ori surveyed with SOXS contains between $\sim 1600$ and $\sim 2350$ stars. The real number is more likely closer to the lower estimate given the caveat noted above. The YSO population is comparable to the ONC population, although it is dispersed over a more extended area and lacks high-mass stars. The XMM-Newton survey has covered only about $60 \%$ of the Spitzer survey in L1641 (Figure 1). We cannot exclude the possibility that a significant fraction of Class III stars are present in the remaining part of the L1641 filament and missed by Spitzer because of a lack of IR excess. The region of L1641 surveyed by Spitzer but not by XMM-Newton contains 22 protostars and 104 Class II YSOs. By assuming the same ratio of Class III to Class II objects found in SOXS, about $365 \pm 20$ YSOs should be in the region without XMM-Newton coverage. The total young population in L1641 is then estimated in the range of 2000-2700 members. If the density of the cloud is correlated with the youth of YSOs embedded in it (Gutermuth et al. 2011), this part of L1641 could contain even more Class III stars relative to Class II objects because of its low density. Further X-ray observations in this region can effectively complete the census of YSOs.

\subsection{X-Ray Spectra and Luminosities}

The analysis of the EPIC spectra provides information about the absorption and the temperature of the emitting plasma.

\subsubsection{Absorption}

The equivalent hydrogen column density, $N_{\mathrm{H}}$, is a way to quantify the absorption of X-rays along the line of sight which is due to atoms and ions. On the other hand, optical and infrared extinction are due to dust grains that have different sizes and shapes. Factors that can affect the $N_{\mathrm{H}} / A_{K}$ ratio among star-forming regions are changes of the metallicity, spatially dependent mass ratio between gas and dust, and variation of the distribution of the grain sizes.

It is possible to estimate the $N_{\mathrm{H}} / A_{K}$ ratio for the X-ray sources with spectral fits and IR counterparts with a known extinction, like $A_{K}$. Our aim here is to compare the extinction $A_{K}$ with

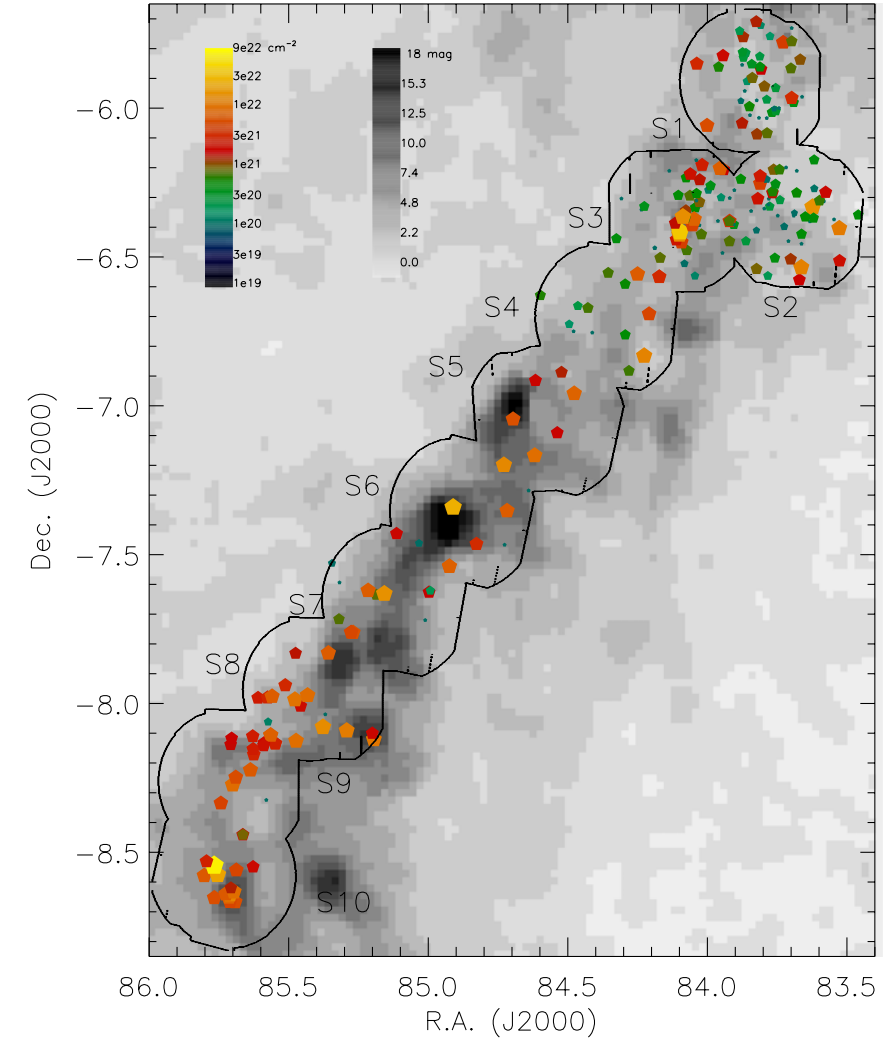

Figure 5. $N_{\mathrm{H}}$ absorption and visual extinction. Symbols are $N_{\mathrm{H}}$ values at the positions of sources with the best fit of the X-ray spectrum. Sizes and colors of the symbols code the intensity of $N_{\mathrm{H}}$ as reported in the scale in the top left corner of the panel. The extinction map is adapted from Figure 4 of Gutermuth et al. (2011). Values of extinction are in the top right scale. Contours and labels refer to the XMM-Newton fields as in Figure 1. Highest values of $N_{\mathrm{H}}$ correspond to more extincted regions. Note the presence of low-absorption stars around $\iota$ Ori and L1641 N.

(A color version of this figure is available in the online journal.)

the absorption values of $N_{\mathrm{H}}$ obtained from the fit to the X-ray spectra. Figure 5 shows a map of $N_{\mathrm{H}}$ values obtained from the best fits to spectra of bright X-ray sources, overplotted on the $A_{V}$ map (adapted from Figure 4 of Gutermuth et al. 2011). Colors and sizes of the symbols are proportional to the $N_{\mathrm{H}}$ column absorption (top left scale). The $A_{V}$ map has been obtained from a map of $(H-K)$ values of Two Micron All Sky 
Survey (2MASS) objects in a rectangular grid with an angular resolution of $\sim 9^{\prime} \times 9^{\prime}$. At each point of the grid, the mean of $(H-K)$ of the 20 nearest objects was calculated, and outliers at $>3 \sigma$ were removed iteratively until convergence was obtained. From the observed $(H-K)_{\text {obs }}$ map, and by assuming a mean intrinsic color $(H-K)=0.13$, the visual extinction map of $A_{V}$ is obtained as: $\left\langle A_{V}\right\rangle=15.87 \times\left(\left\langle(H-K)_{\text {obs }}\right\rangle-0.1\right)($ Lada et al. 1994; Lombardi \& Alves 2001). As Gutermuth et al. (2005) warned, this map can be biased toward lower absorption values than the total cloud value for the line of sight because of the presence of stars embedded in the cloud itself.

In the northern part of the map, around $\iota$ Ori, we observe a mix of very absorbed stars with $N_{\mathrm{H}}>10^{21} \mathrm{~cm}^{-3}$ (red and orange dots in Figure 5) and less absorbed stars that appear to be in front of the cloud (green symbols in the same figure). This is evidence of a more evolved population of PMS stars around $\iota$ Ori, not embedded in the cloud but rather in the foreground with respect to the ONC (Bally 2008; Alves \& Bouy 2012). The less absorbed stars in the northern part of L1641 are part of this older population which sits in front of the cloud or at its surface where the absorption is lower, with ` Ori being the most massive member of this foreground cluster. On the other hand, in the southern part of L1641, strongly absorbed stars are a major fraction of the whole population. The highest values of $A_{V}$ are found in L1641 S, where high $N_{\mathrm{H}}$ absorption values are also measured.

We evaluate the relationship between $N_{\mathrm{H}}$ absorption and $A_{K}$ extinction, using the $A_{K}$ values from the individual stars themselves assuming the extinction law from Flaherty et al. (2007). A linear relation between $N_{\mathrm{H}}$ and $A_{K}$ (with $A_{K} \sim$ $0.1 A_{V}$; Rieke et al. 2004; Rieke \& Lebofsky 1986) yields values of $N_{\mathrm{H}} / A_{K}$ in the range $1.8-2.2 \times 10^{22} \mathrm{~cm}^{-2} \mathrm{mag}^{-1}$ for the interstellar medium (ISM; Vuong et al. 2003; Gorenstein 1975). For other SFRs, Feigelson et al. (2005) and Vuong et al. (2003) have found the slopes slightly lower $\left(N_{\mathrm{H}} / A_{K}=1.6 \times\right.$ $10^{22} \mathrm{~cm}^{-2} \mathrm{mag}^{-1}$ ), while significantly lower values have been found by Winston et al. (2010) for the Serpens and NGC 1333 SFRs. An intermediate slope of $1.3 \times 10^{22} \mathrm{~cm}^{-2} \mathrm{mag}^{-1}$ is found in the region of IRAS 20050+2720 (Günther et al. 2012).

Figure 6 shows the scatter plot between the column absorption values $N_{\mathrm{H}}$ and $A_{K}$. The linear fit of all points gives $N_{\mathrm{H}}=(7.0 \pm 0.3) \times 10^{21} A_{K}$; by excluding YSOs with IR excesses we obtain $N_{\mathrm{H}}=(7.6 \pm 0.5) \times 10^{21} A_{K}$, still consistent with the value of the whole sample. When excluding objects with IR excess, we reduce the influence of local absorption due to the presence of the disk in Class II YSOs and the envelope in Class I YSOs. Our best-fit value is similar to those obtained by Winston et al. (2010) for Serpens and NGC 1333 - none of which contain high-mass stars, and it is lower than those found for high-mass star-forming regions such as RCW 38, RCW 108 (Wolk et al. 2006, 2008), and the ONC as well as the ISM (Vuong et al. $2003 ; N_{\mathrm{H}} / A_{K}(\mathrm{ONC})=(1.0 \pm 0.1) \times 10^{22} \mathrm{~cm}^{-2} \mathrm{mag}^{-1}$; $\left.N_{\mathrm{H}} / A_{K}(\mathrm{ISM}) \sim(2.0 \pm 0.2) \times 10^{22} \mathrm{~cm}^{-2} \mathrm{mag}^{-1}\right)$.

If we consider the $N_{\mathrm{H}} / A_{K}$ ratio as a measure of the gas-to-dust ratio, IRAS 20050+2720, Serpens, and NGC 1333 are dust-rich (or gas-poor), compared with the ISM. In contrast, the sample from Günther \& Schmitt (2008) shows most objects to be dustdepleted (or gas-enriched) with much lower absolute values for the extinction. These sources are mostly from the Taurus-Auriga molecular cloud complex, which is more dispersed and older than IRAS 20050+2720, Serpens, and NGC 1333, and the SFR contributes little to the line-of-sight extinction. The extinction in Serpens, IRAS 20050+2720, and NGC 1333 should be

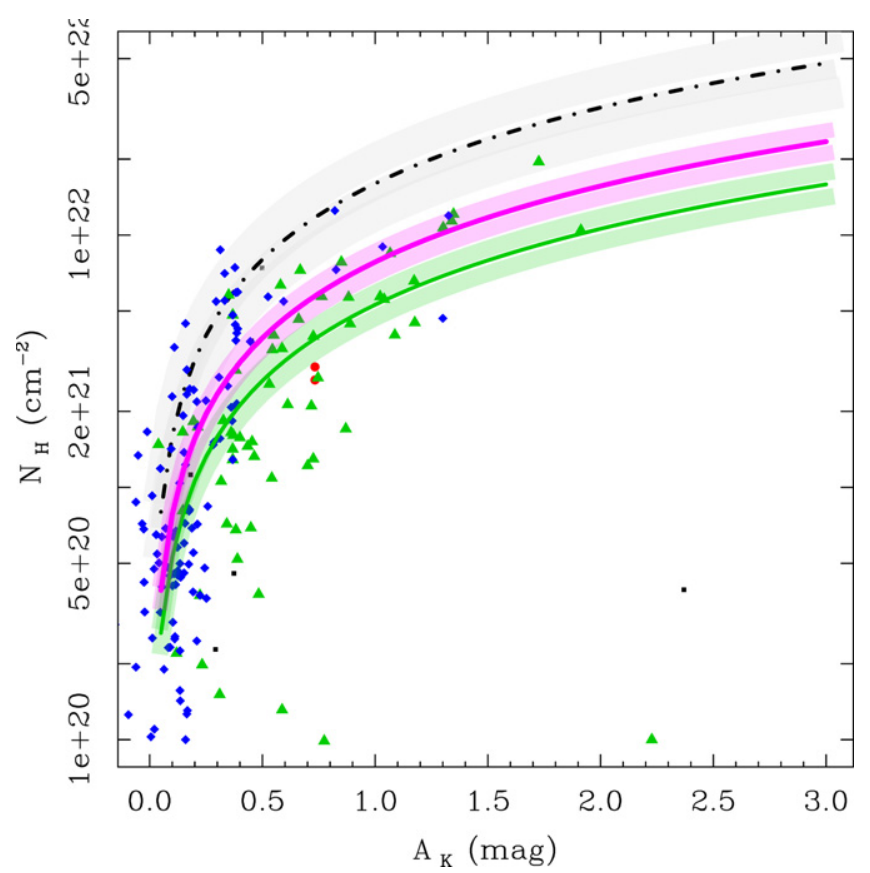

Figure 6. Scatter plot of $N_{\mathrm{H}}$ vs. $A_{K}$ values. Red circles are protostars, green triangles are Class II objects, and blue diamonds are Class III candidates. Black dots are other objects not classified as PMS stars. The dot-dashed line is the relation derived for the ISM by Vuong et al. (2003). Solid lines are the best fits to all data (green line) and only objects without IR excess (magenta line): $N_{\mathrm{H}} \sim(0.70 \pm 0.03) \times 10^{22} \cdot A_{K}$ and $N_{\mathrm{H}} \sim(0.76 \pm 0.05) \times 10^{22} \cdot A_{K}$, respectively. Shaded areas represent the $90 \%$ level of uncertainty of the slope values. The $N_{\mathrm{H}} / A_{K}$ ratio in L1641 is different than that in the ISM but consistent with those found for the NGC 1333 and Serpens clusters.

(A color version of this figure is available in the online journal.)

dominated by local circumstellar material, which could be dustdepleted due to the energy input from the star, gravitational settling of grains in the disk-midplane, and planet formation. However, in the younger star-forming regions, we mostly see the cloud material, which could be dust-rich for those clouds without high-mass stars, or metal-poor in the gas phase due to grain formation.

While gas depletion is a possibility, it is not the only possible cause of the anomalously low $N_{\mathrm{H}}$ to $A_{K}$ ratio. Current models predict that volatiles accrete on grains in cold dense clouds, and the grains start sticking together so that growth occurs with time (Ormel et al. 2011). Evidence for grain growth in molecular clouds has been found in the changing IR extinction law toward the high extinction line of sight (Flaherty et al. 2007; Chapman et al. 2009; McClure 2009; Chiar et al. 2007). Recent submillimeter observations made with Herschel by Roy et al. (2013) provide evidence for a change in the dust opacity which is explained as a result of the evolution of dust grains.

In contrast, without the condensation of volatiles, the grains reach an equilibrium size quickly by balancing aggregation and fragmentation. Thus, we would expect the $N_{\mathrm{H}} / A_{K}$ ratio to decrease with time in cold clouds until the clouds are heated to the point that the volatiles evaporate. The survey of L1641 shows that the $N_{\mathrm{H}} / A_{K}$ ratio is changing within the Orion A cloud; the ONC shows a $N_{\mathrm{H}} / A_{K}$ more typical of the ISM and other highmass star-forming regions, while the $\mathrm{L} 1641$ region shows a ratio similar to that of dense molecular clouds forming clusters of low to intermediate stars such as Serpens and NGC 1333 (Winston et al. 2010). We hypothesize that the difference between the massive and low- to intermediate-mass star-forming regions is 
the heating of the dust by the young stars. In the clusters of low- to intermediate-mass stars, the inner region of the clouds is shielded from the interstellar radiation field and there is relatively little heating from the embedded stars. In contrast, toward massive star-forming regions, the intense radiation fields from the high-mass stars heat the dust. The heating of the dust raises the temperature of the grains, causing the evaporation of volatiles and potentially leading to the disruption of the coagulated dust grains. The gas kinetic temperatures in cores toward the Orion cloud show a large drop between the ONC region and the L1641 region, supporting our hypothesis.

\subsubsection{Temperatures}

Given the low counts of our spectra and their low resolution, a detailed analysis of the thermal components of the emitting plasma is not possible. Nevertheless, a rough analysis is still possible to differentiate between hot or cold spectra. The spectra with highest count rates were modeled with a two-component thermal model. In these 40 cases (cf. Table 3), we obtained a representative mean temperature by calculating the weighted mean of the two temperatures, the weights being the values of emission measures obtained for each component in order to compare with single temperature best-fit models.

Class I objects appear hotter on average than Class II and Class III objects. The median plasma temperatures are: $k T=1.8 \pm 0.7 \mathrm{keV}$ (Class I), $1.2 \pm 0.6 \mathrm{keV}$ (Class II), $0.9 \pm 0.3$ $\mathrm{keV}$ (Class III candidates). The absorption due to the gas around Class I and Class II objects can mimic hotter spectra since the presence of any soft component below $1 \mathrm{keV}$ is heavily reduced. Similar differences in temperatures are also observed in the Rho Ophiuchi forming region (Pillitteri et al. 2010). In some cases, the spectra of YSOs in the Ophiuchus molecular cloud show a $6.7 \mathrm{keV}$ line from highly ionized $\mathrm{Fe}$ atoms and this is evidence of very hot plasma $(k T \geqslant 5 \mathrm{keV})$. In SOXS spectra we do not detect this feature, either because of the lower count statistics or because of an intrinsically cooler temperature. As a consequence, we cannot disentangle the effects of a genuinely high temperature from those related to strong absorption.

\subsubsection{X-Ray Luminosities}

For the brightest sources, the X-ray luminosities of classified PMS stars have been calculated based on fluxes directly determined from the best-fit modeling. For the remaining YSOs, faint in X-rays, we have used the PIMMS software to determine a count rate to flux CF. As discussed before, the values of absorption and plasma temperatures differ, on average, among the different SED class samples. For each sub-sample (protostars, Class II, and Class III sources), we used a thermal APEC 1-T model, with $N_{\mathrm{H}}$ absorption and plasma temperature corresponding to the median of values derived from the spectral analysis of bright spectra with the XSPEC program. ${ }^{12}$

We compare the cumulative distributions of X-ray luminosities (CDXLs) of protostars, Class II objects, and Class III candidates in the regions around $\iota$ Ori (field $S 1$ ), L1641 North (fields $S 2+S 3+S 4$ ), and L1641 South (fields $S 7+S 8+S 9+S 10$ ).

\footnotetext{
12 Another approach that we tried was to estimate the absorption for faint sources from the relationship between the median energy of their spectra and $N_{\mathrm{H}}$. Feigelson et al. (2005) showed that for Chandra-ACIS COUP data this can be calibrated from the spectral analysis of bright sources. However, an attempt to use this method revealed that the relationship in $X M M-N e w t o n-E P I C$ is steeper than in Chandra-ACIS due to the different responses. For median energies below $1.3 \mathrm{keV}$ and a range of $N_{\mathrm{H}}=10^{19}-3 \times 10^{21} \mathrm{~cm}^{-2}$ this method is thus ineffective.
}

Table 8

Median Values (and Mean Absolute Deviations, MAD, in Parentheses) of $\log L_{X}$ of YSOs Separated by SED Class and Region

\begin{tabular}{lcccc}
\hline \hline \multirow{2}{*}{ Regions } & \multicolumn{4}{c}{ Classes } \\
\cline { 2 - 5 } & Class I & Class II & Class III & Class II+III \\
\hline All & $29.84(0.56)$ & $29.69(0.67)$ & $29.57(0.63)$ & $29.60(0.64)$ \\
$\iota$ Ori & {$[30.23]$} & $30.06(0.58)$ & $29.75(0.51)$ & $29.77(0.58)$ \\
L1641 N & $29.92(0.54)$ & $29.58(0.56)$ & $29.50(0.63)$ & $29.51(0.58)$ \\
L1641 S & $29.74(0.09)$ & $29.72(0.81)$ & $29.50(0.69)$ & $29.55(0.71)$ \\
\hline$N 1+N 2+N 3$ & $29.16(0.70)$ & $29.93(0.60)$ & $30.00(0.59)$ & $29.96(0.60)$
\end{tabular}

Notes. MADs are calculated as in R-language implementation, and are scaled for the factor 1.4826 which ensures consistency with $\sigma$ of a normal distribution. In the $\iota$ Ori field we detected only one Class I object.

Class III candidates are selected based on their X-ray emission and we do not have complete membership information for undetected Class III objects. Furthermore, a few foreground stars are expected in this sample. In Section 4.1.4, we will calculate the X-ray luminosity function (XLF) for the sub-sample of YSOs that have membership and youth information obtained with additional optical spectroscopy and thus we can also consider those YSOs undetected in X-rays.

In Figure 7, we show the CDXLs of detected YSOs separated by SED classification and by regions. In Table 8 , we report the median and the mean absolute deviation (MADs) of the luminosities for the YSOs separated by SED class and zones. The panels in the top row of Figure 7 comprise all the detections separated by SED class only. For comparison, we also plot a log-normal model of the XLF similar to that of Feigelson et al. (2005) from COUP data (solid black line). We use this model $\left(\mathrm{XLF}_{\mathrm{COUP}}\right)$ to roughly fit the high-luminosity tail of the CDXLs down to $\log L_{X} \sim 29-29.5$. Below this threshold, the number of undetected sources should increase significantly, and the observed CDXL deviates from the log-normal model. Regarding the adoption of the $\mathrm{XLF}_{\mathrm{COUP}}$, a note of warning has to be added. While the existence of a universal XLF was initially proposed by Feigelson et al. (2005), subsequent studies have shown that the modeling and the slope of the high-luminosity tail of the distribution can depend on the masses of stars, their distribution, and their age with respect to the COUP sample (Getman et al. 2006; Wang et al. 2008; Kuhn et al. 2010). The modeling of the XLD of COUP has been changed: Getman et al. (2006) used a broken power law to model the lightly obscured YSOs in the COUP data set, while in other SFRs a log-normal model gave a good fit to the XLDs. Prisinzano et al. (2008) have calculated mass-stratified XLFs from the COUP data for different SED classes, adopting a log-normal model. Among Class II and Class III YSOs with masses in $0.1-0.9 M_{\odot}$, they find similar medians but different widths of XLFs, with the Class III having a narrower distribution of luminosities (Figure 9 in Prisinzano et al. 2008).

We have used the same median of $\mathrm{XLF}_{\mathrm{COUP}}\left(\log L_{X}=29.3\right)$ but we have changed the normalization and adjusted the standard deviation to the range $\sigma=0.7-0.8$ for an improved fit. The narrower distribution suggests an intrinsic difference between L1641 and the ONC, perhaps due to the lack of high-luminosity massive stars (Hsu et al. 2012). A similar result has been observed by Günther et al. (2012) in an analysis of the low-mass star-forming region IRAS 20050+2720. The CDXLs of Class I, Class II, and Class III objects are in reasonable agreement with the log-normal model for $\log L_{X} \gtrsim 29$. 

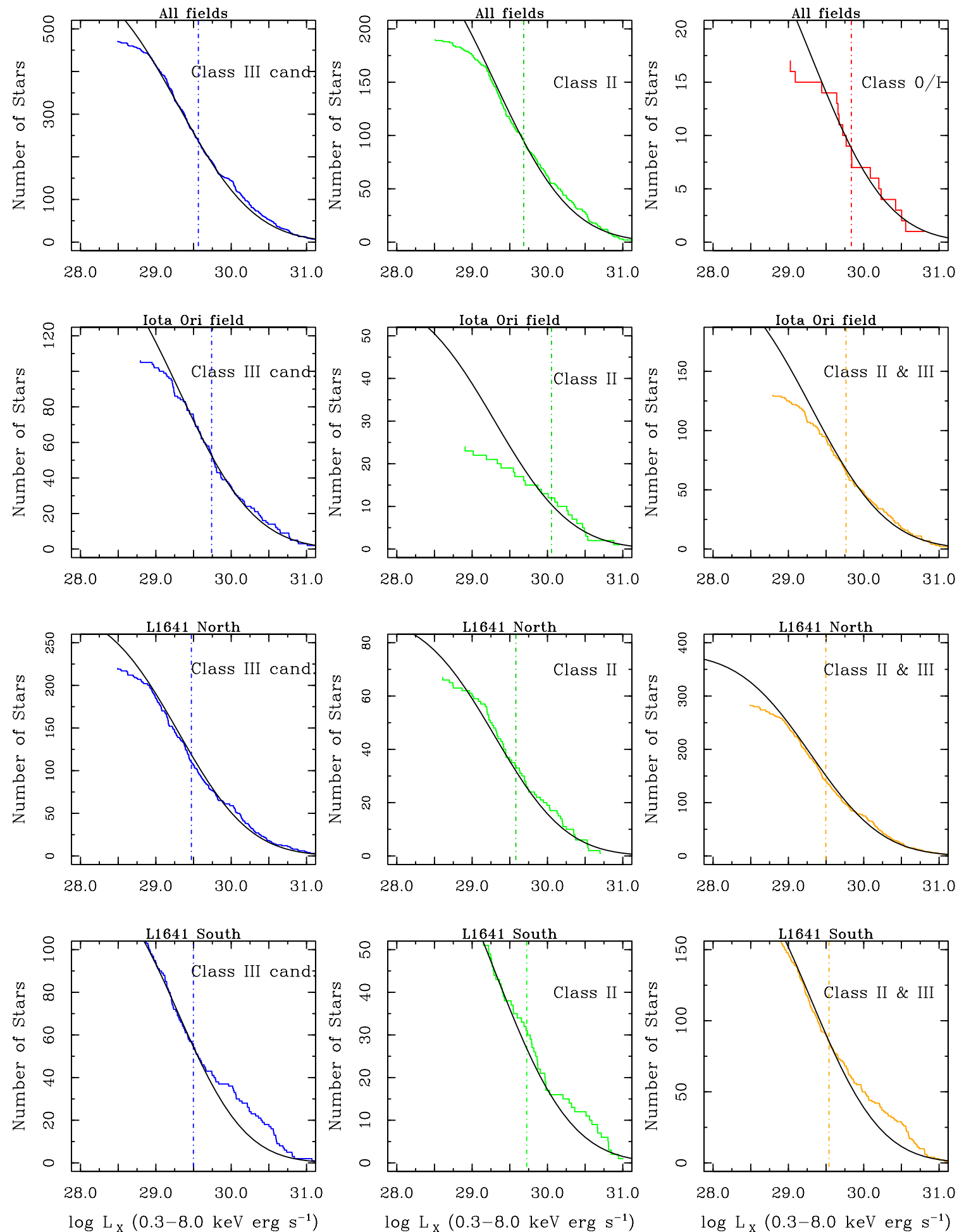

Figure 7. Cumulative distributions of X-ray luminosities (CDXLs) of Class I (red), Class II YSOs (green) and Class III candidates (blue). The top panel shows the curves for each sub-sample, whereas the other three panels show the curves for sub-regions: field of $\iota$ Ori, L1641 N, and L1641 S. Vertical colored lines are the medians of the distributions. For each region we have also considered the CDXLs of Class III candidates and Class II objects. The agreement with the model curve (black solid curve) is good in general down to $\log L_{X} \sim 29.5$. The deviation from the model curve is observed in L1641 $\mathrm{N}$ around $\log L_{X} \sim 30$ and suggests that there is a sub-sample of stars closer than the ONC.

(A color version of this figure is available in the online journal.)

Protostars are slightly more luminous than Class II and Class III candidates (panels in top row of Figure 7). This overluminosity is likely due to a selection bias because protostars are highly embedded X-ray sources that suffer from higher absorption and extinction when compared to Class II and Class III objects. The high number of undetected protostars (124 out of
147) means that we have detected only those that are exceptionally bright and hot in X-rays. The sources with fainter and/or cooler spectra are too absorbed to be detected in X-rays by our survey.

A comparison of CDLXs and the values of the medians of the $\log L_{X}$ of Class III candidates shows that around $\iota$ 


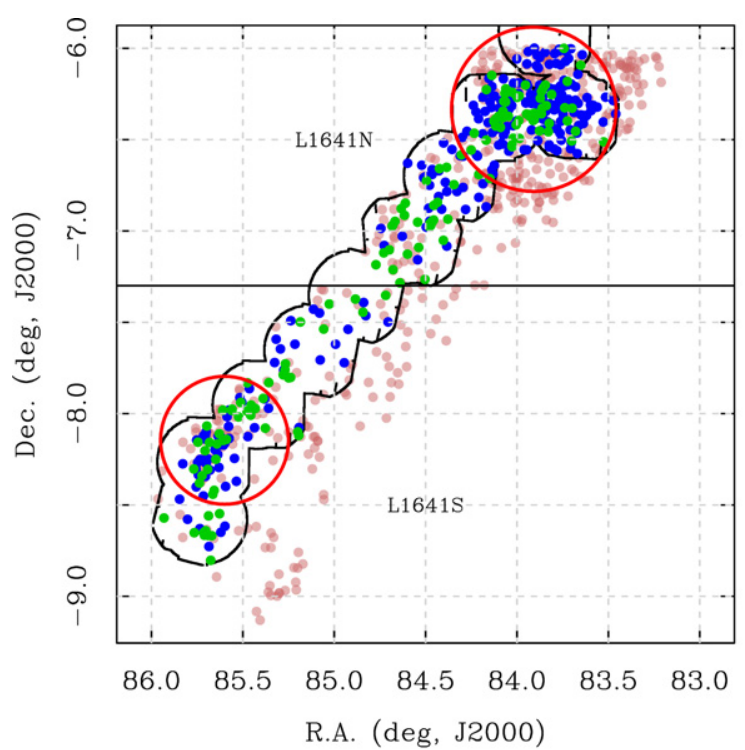

Class III members

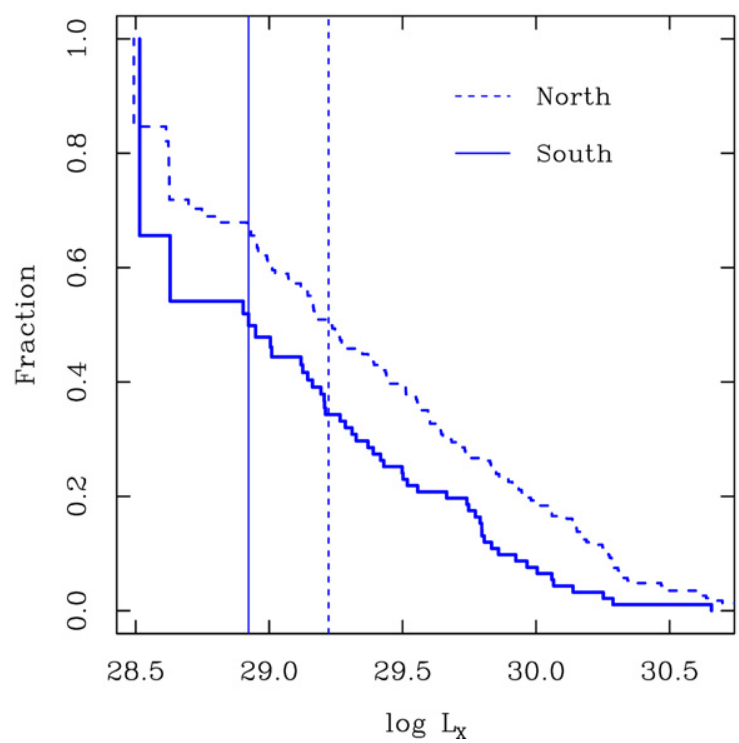

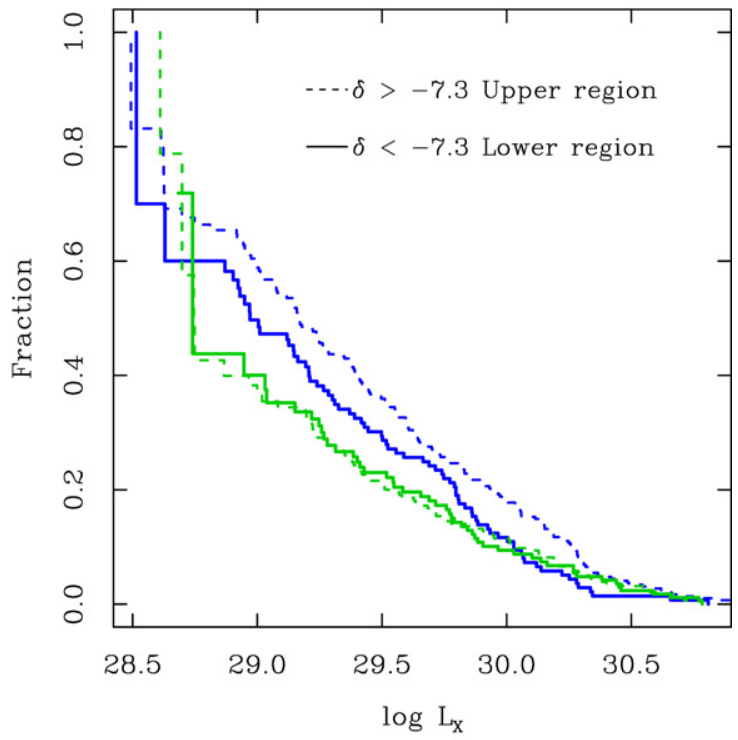

Class II members

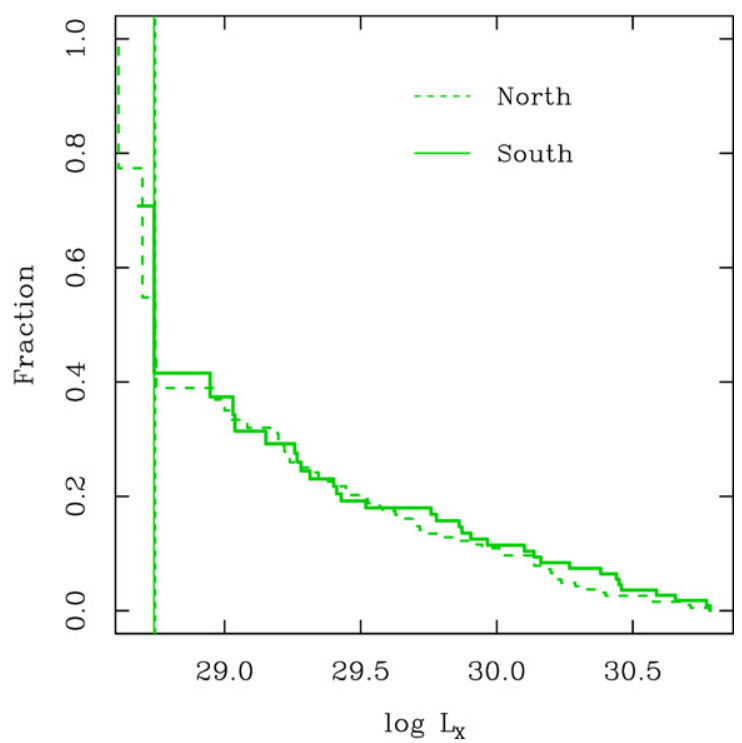

Figure 8. Top left panel: positions of the spectroscopic members from Hsu et al. (2012) (red symbols), matched with Class III candidates (blue points) and Class II objects (green). Horizontal lines and circles mark the sub-regions used to divide the stars into L1641 N and L1641 S and their core regions. Top right: XLFs of Class II (green) and Class III stars (blue) for stars north (dashed line) and south (solid line) to $\delta=-7.3$. Bottom panels: XLFs for Class III (left) and Class II objects in the cores of L1641 N (dashed line) and L1641 S (solid line). Vertical lines mark the medians of XLFs for the South (solid line) and North (dashed line) cores. A difference in the XLFs is markedly visible only between Class III stars belonging to L1641 S and L1641 N.

(A color version of this figure is available in the online journal.)

Ori Class III candidates are systematically more luminous (by 0.25 dex) than the Class III candidates L1641 S and L1641 N (Figure 7, first column plots, and Table 8, third column). A Kolmogorov-Smirnov test gives a probability of $<0.5 \%$ for the two distributions being drawn from the same parent distribution. The same is not evident from the comparison of the CDLXs and the median $\log L_{x}$ of Class II objects in the same regions.

We observe an excess around $\log L_{X} \sim 30 \mathrm{erg} \mathrm{s}^{-1}$ in the CDLX of Class II and Class III objects in L1641 N with respect to the log-normal curve. These differences can be ascribed to the presence of two groups of stars at two different distances. Around $\iota$ Ori, and to some extent in L1641 N, there is a population of older and more evolved Class III stars at a closer distance than the ONC and L1641. The fraction of Class II objects belonging to this cluster is much lower than in the ONC. The presence of this closer cluster causes a small excess of CDLXs of Class II of $\iota$ Ori and L1641 N, but produces the 0.25 dex higher luminosity in Class III sample around $\iota$ Ori. In the following section (Section 4.1.4), we make use of spectroscopic membership information to confirm this result.

\subsubsection{X-Ray Luminosity Functions of Spectroscopic Members}

Hsu et al. (2012) published a spectroscopic survey of stars in the L1641 filament. By using $\mathrm{H} \alpha$ emission and $\mathrm{Li}$ absorption at $6708 \AA$ they classified 864 stars as members from a list of objects taken from the Spitzer catalog (Megeath et al. 2012). They suggested another 98 probable members of the region, and estimated that the total population in L1641 is on the order of 1600 stars, which is consistent with our estimate. The area surveyed by Hsu et al. (2012) covers the region observed with Spitzer (cf. right panel of Figure 1) while the XMM-Newton observations cover only the more active eastern half of the 
Spitzer survey of L1641. Nonetheless, 652 out of the 864 members $(\sim 75 \%)$ fall in the SOXS field of view. Hsu et al. determined that 406 members have IR excess while 458 do not. We have considered only the conservative sample of $406+458$ members from Hsu et al. (2012) in the following analysis. We have matched our list of Class III candidates and Class II objects detected in X-rays against the list of 864 spectroscopic members in order to determine their XLFs more precisely.

The XLFs were computed with a version of the ASURV software (Lavalley et al. 1992) to take into account the upper limits of undetected members. For the members not matched with the Hsu et al. catalog and within the SOXS fields, we have calculated upper limits to the X-ray luminosity. Figure 8 shows the positions of the matches between our catalog of Class II and Class III candidates and the Hsu et al. (2012) list of members (top left panel). The circles show the core regions of L1641 North and South. The same figure shows the XLFs of the stars divided roughly into two groups, above and below $\delta=-7.3$ (top right panel), as well as those in the cores of L1641 North and South, respectively (bottom panels). We have divided the stars into two spatial groups to test systematic differences between the northern and the southern parts. The Class III stars exhibit a large difference in X-ray luminosity when comparing those above and below $\delta=-7.3$, and the difference is even more marked when considering only the core regions with the highest stellar density in L1641 North and South. Only Class III stars exhibit such a strong difference in the respective XLFs. Class II objects have a similar XLF in both spatial subgroups. The difference of medians of the XLFs of the cores between L1641 N and $\mathrm{L} 1641 \mathrm{~S}$ is $\sim 0.3 \pm 0.1 \mathrm{dex}$. When compared with the XLFs of Prisinzano et al. (2008) in the same range of masses, we observe that, the medians for the XLFs of the Class III stars around $\iota$ Ori are lower than those reported by Prisinzano et al. of $\sim 0.3$ dex. The stellar masses of the members of the Hsu et al. catalog with $\mathrm{X}$-ray detection are in the range $0.1-0.6 M_{\odot}$, which is one of the mass-strata defined by Prisinzano et al. (2008) to calculate their XLFs from the COUP data set. The difference among Class III stars is best explained by arguing that there is a cluster of Class III stars in L1641 N closer than the ONC. The fact that Class II objects do not show the same difference implies that the closer cluster is composed mainly of stars older and more evolved than those in the ONC but still in the PMS phase. The presence of a cluster in the foregound of Orion+A was also proposed by Alves \& Bouy (2012), on the basis of infrared photometry and optical spectroscopy of YSOs in Orion A.

\subsection{Comparison with the ONC}

We have analyzed the observations available in the $X M M$-Newton archive and listed them in Table 1 in order to compare the X-ray emission in L1641 with that of the young stars around the densest part of the ONC. The core of the ONC is located between fields $N 2$ and N3. Figure 9 shows the positions of protostars, Class II objects, and Class III candidates in the northern fields. A strong alignment of protostars in a filament running along the three northern fields is evident. Also, the Class II objects are especially concentrated around the core of the ONC, while Class III candidates are more spread out. The ratio between the number of Class III candidates and Class II objects detected in X-rays decreases from north to south. This ratio is $\sim 2.2,0.9$, and 0.52 in the $N 1, N 2$, and $N 3$ fields, respectively. The increasing number of Class II objects with respect to the number of diskless stars can be understood as an evolu-

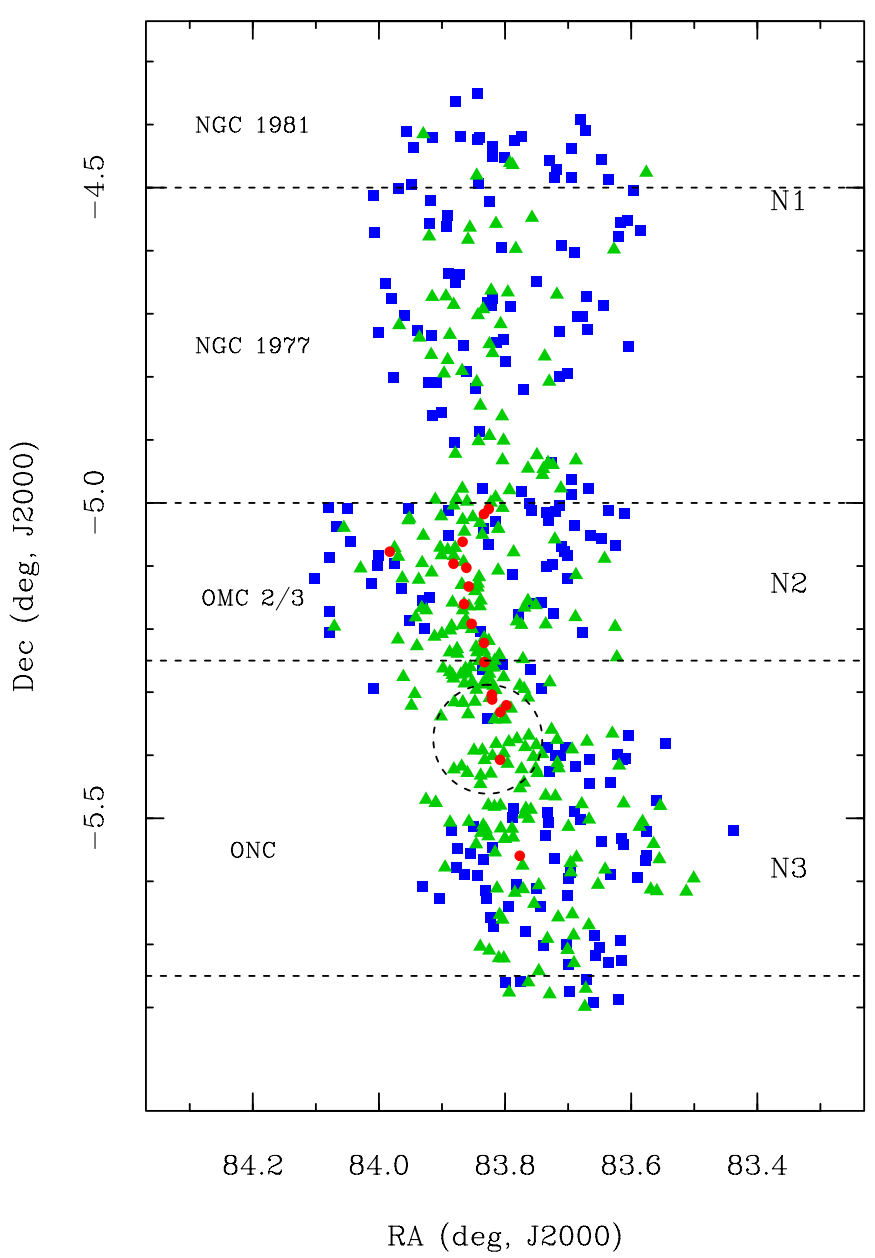

Figure 9. Map of the YSOs detected in X-ray in the northern fields $N 1, N 2, N 3$ around ONC. We marked with colored large symbols the sources matched with Spitzer YSOs (Megeath et al. 2012). Red circles are protostars, green triangles are Class II stars, and blue squares are Class III candidates. The main sub-regions are separated by horizontal lines. The crowded region excluded from X-ray data is shown with a dashed circle. Class II objects and protostars preferentially lie along the gas filament, while the Class III candidates are more spread out.

(A color version of this figure is available in the online journal.)

tionary effect because younger and less evolved stars are found closer to the core of the ONC, while more evolved stars are spread to larger distances.

We have derived X-ray luminosity distributions (CDLXs) for $\mathrm{X}$-ray-detected Class II and Class III candidates and compared these with those of L1641 YSOs. We have excluded the sources located near the core of the ONC in a circle of center R.A. $05^{\mathrm{h}} 35^{\mathrm{m}} 19.3$, decl. $-05^{\mathrm{d}} 21^{\mathrm{m}} 47^{\mathrm{s}} .6$ and radius 5.2 that corresponds to $0.6 \mathrm{pc}$ at the distance of the $\mathrm{ONC}$, and encompassing its densest part (marked in Figure 10). This is a region with many confused and blended sources in the XMM-Newton image (Figure 2), and the instrument has a less calibrated PSF because it is at the edge of the field of view. Figure 10 shows the XLFs of Class II objects and Class III candidates detected in the northern fields $N 1, N 2$, and $N 3$.

From the fits of CDLXs to the log-normal model, we infer a normalization of 550 stars for the sample of Class II objects and Class III candidates. This, combined with the number of detections in each sample (235 and 211 objects), suggests a rate of detection of $38 \%-42 \%$ and thus a total population of $\sim 1200 \pm 100$ YSOs in these fields (excluding the core of the ONC). 

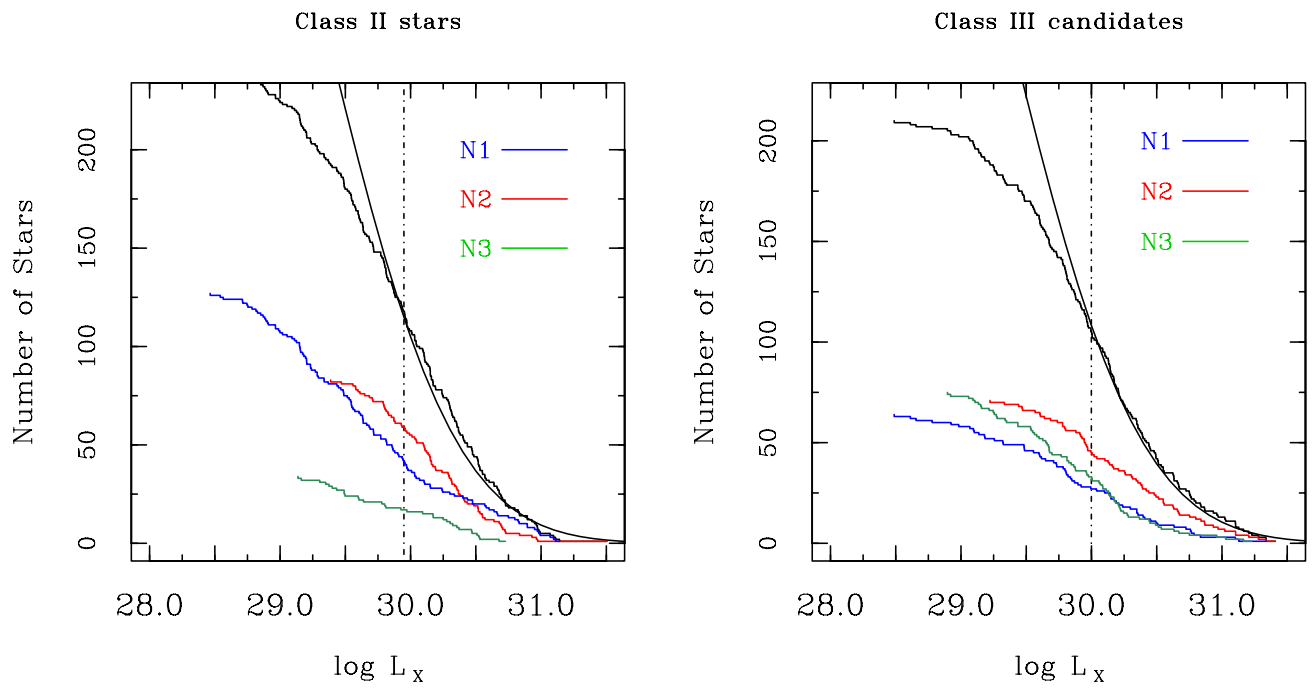

Figure 10. XLFs of Class II objects and Class III candidates in the northern fields as in Figure 7. The solid stepped curve is the total sample; colored curves are the samples in the three northern fields as indicated by the labels. The dot-dashed vertical lines mark the medians of the total sample of Class II and Class III stars. The smooth curve is the model distribution.

(A color version of this figure is available in the online journal.)

The number of detected Class II stars (235) is slightly larger than that of Class III objects (211). The flatter slope of Class III CDLXs at low luminosities suggests that a fraction of Class III objects could be undetected in this range of luminosities. Hence the ratio of Class III to Class II objects could be close to 1, but still lower than around $\iota$ Ori (cf. Table 7). Interpreting this ratio as an evolutionary tracer, it suggests that this group of stars is younger than the stars around $\iota$ Ori.

\subsection{Spatial Distribution of PMS Stars}

In this section, we give a description of the spatial distribution of the young stellar population around ` Ori and in L1641, based on the classifications of YSOs obtained with Spitzer and $X M M-N e w t o n$ data. The analysis in Section 4.1.1 shows that, on average, Class III objects are found in less absorbed regions (see Figure 5) and that they could be a slightly intrinsically more X-ray luminous than Class II objects (see also Preibisch et al. 2005). This can lead to an easier detection of Class III stars with respect to Class II objects in X-rays. Figure 11 shows the positions of the Class III candidates (left panel) and Class II and protostars detected in X-rays (right panel). In both samples a dominant clustering of YSOs appears around $\iota$ Ori and near L1641 S, while the central region of L1641 is relatively less populated.

To address the question of how clustered the YSOs in our survey are, we use the distributions of distances to the nearest neighbor (NN distances) and Monte Carlo simulations. For each object in each sample of Class II and Class III YSOs, we calculated the distance to the closest object and we obtained a cumulative distribution of distance greater than a certain value, and normalized for the total number of objects in that sample. We obtained analogous distributions for the ONC, the upper and lower regions of L1641, their cores as defined in Section 4.1.4, and the ONC. These distributions are shown in Figure 12. The number of objects in each sample can bias the comparison of the NN distributions. We have scaled the NN distances of the Class II samples to take into account the different numbers of objects in the compared samples.

The comparison of the observed distribution and the envelope of the simulated distributions of NN distances will show any differences with respect to a uniform distributions of stars, and quantify any local clustering of objects within a spatial scale significantly smaller than the surveyed area. For this purpose, we ran a set of Monte Carlo simulations of uniformly distributed stars with size equal to the total sample of L1641 Class II and Class III objects and that of the ONC samples. The curves of observed NN distances are not fit by a Gaussian model. Rather, a linear fit to the cores of the distributions and their wings, similar to the approach followed by Gutermuth et al. (2009), allows us to estimate a characteristic separation within which most of the objects are grouped.

In the ONC, the distributions of NN distances show a concentration of objects at $d<0.2$ pc while L1641 shows the bulk of NN distances within $0.3 \mathrm{pc}$ (top panels in Figure 12). NN distances of Class II and III objects in L1641 are marginally consistent with a uniform distribution. These are more discrepant at short distances, while the distribution of objects further out more closely follows a uniform distribution. At NN distances shorter than $0.15 \mathrm{pc}$ the agreement with the uniform distribution is at level of $\leqslant 1 \%$, thus suggesting that the stars in both samples of Class II and III objects are spaced more closely than expected from a uniform distribution. This is even more evident in the sub-samples of the upper/lower regions of L1641 and in their respective cores (see Figure 8 for the definition of cores). Class III stars in the lower region and the southern core are markedly different from a uniform distribution, while their distribution in the northern half of L1641 and the northern core is indistinguishable from a uniform distribution. The distributions in the lower region as well as the southern core appear to be bi-modal, with a minor fraction of clustered sources with separation $<0.15 \mathrm{pc}$, surrounded by a more dispersed population. The northern region shows a different situation, with the stars densely packed and their distribution is not too different from a uniform population. This can be seen as a bias of the X-ray survey, because the actual spatial scale of the cluster of Class III stars toward $\iota$ Ori and L1641 $\mathrm{N}$ is of the same scale or larger than the XMM-Newton field of view, hence the stars are almost uniformly distributed within the field of view.

The presence of clustering in the two core fields can also be inferred by the typical separations of the Class II objects and 

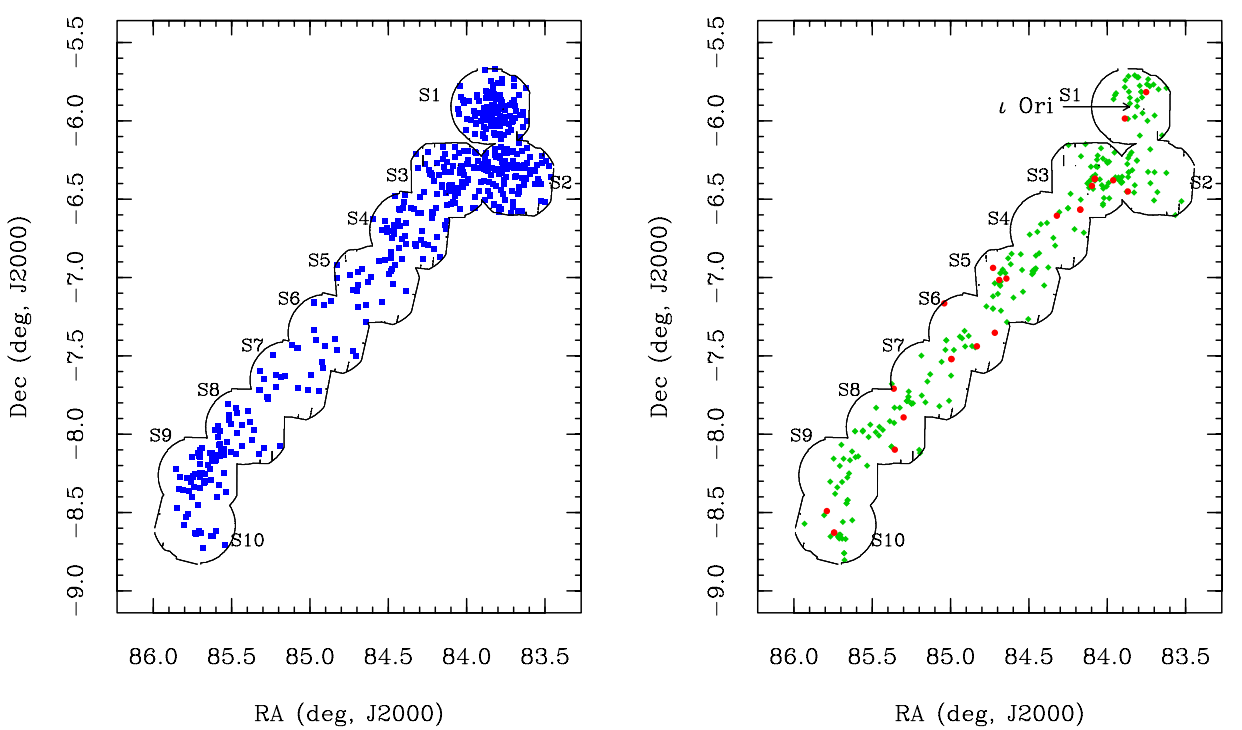

Figure 11. Distribution of Class III candidates (left panel) and Class II YSOs detected in X-rays (right panel, green symbols) toward L1641. Red filled circles in the right panel are the protostars detected in X-rays. Class III candidates are grouped in two main sub-clusters, one around $\iota$ Ori and L1641 S. Class I and Class II objects are detected along the gas filament and less spread out than Class III candidates.

(A color version of this figure is available in the online journal.)
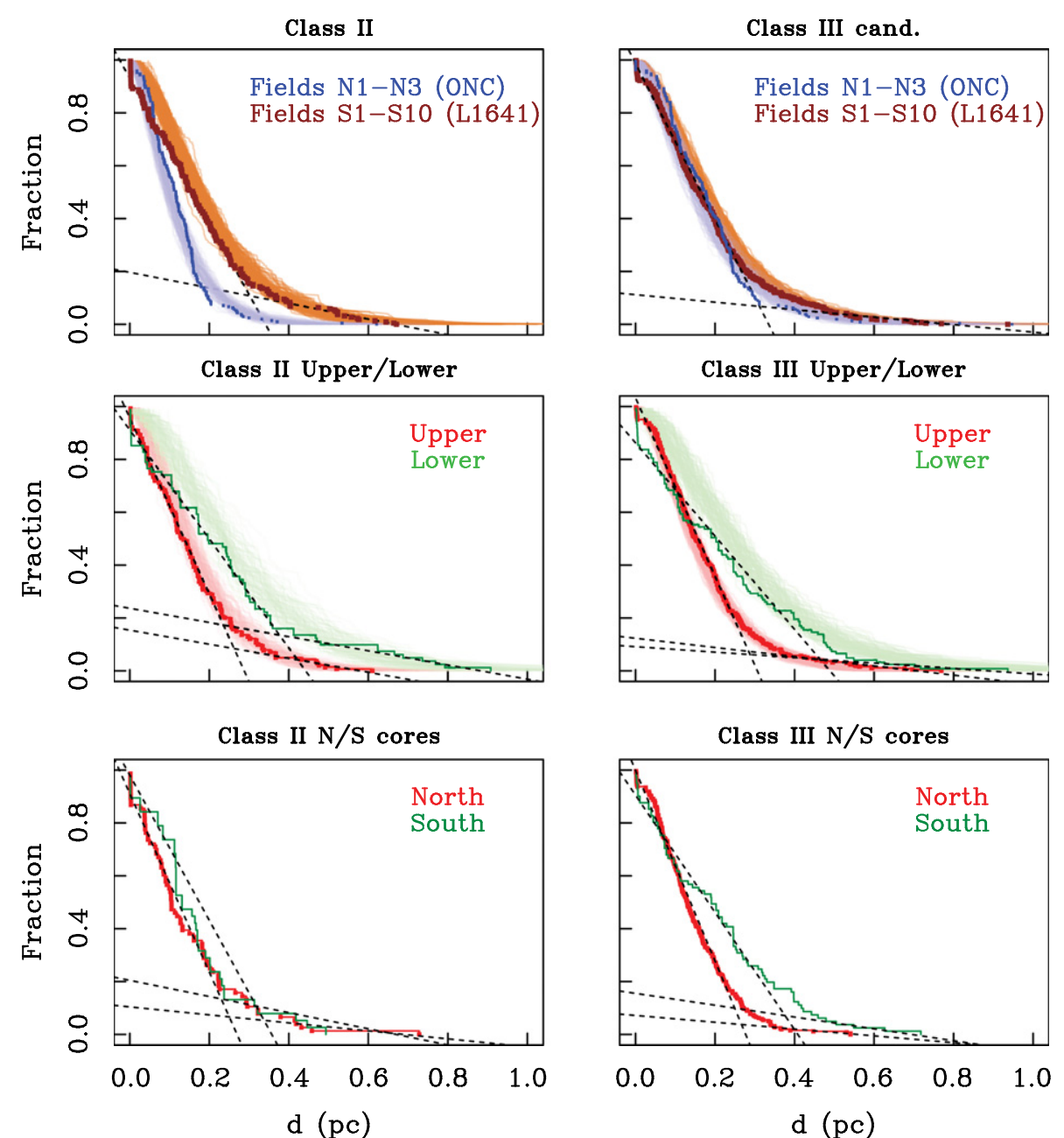

Figure 12. Fractional cumulative distributions of nearest neighbor distances of Class II objects and Class III candidates. Top panel: distributions for the samples of Class II objects (left panel) and Class III candidates (right panel) in L1641 and ONC. Central and bottom panels are the same for the samples in the upper and lower regions of L1641 and their cores. Dashed lines are the linear fits to the cores and the tails of the distributions. Shaded areas show an area encompassing the 10,000 Monte Carlo simulations of uniform distributions.

(A color version of this figure is available in the online journal.) 
Class II

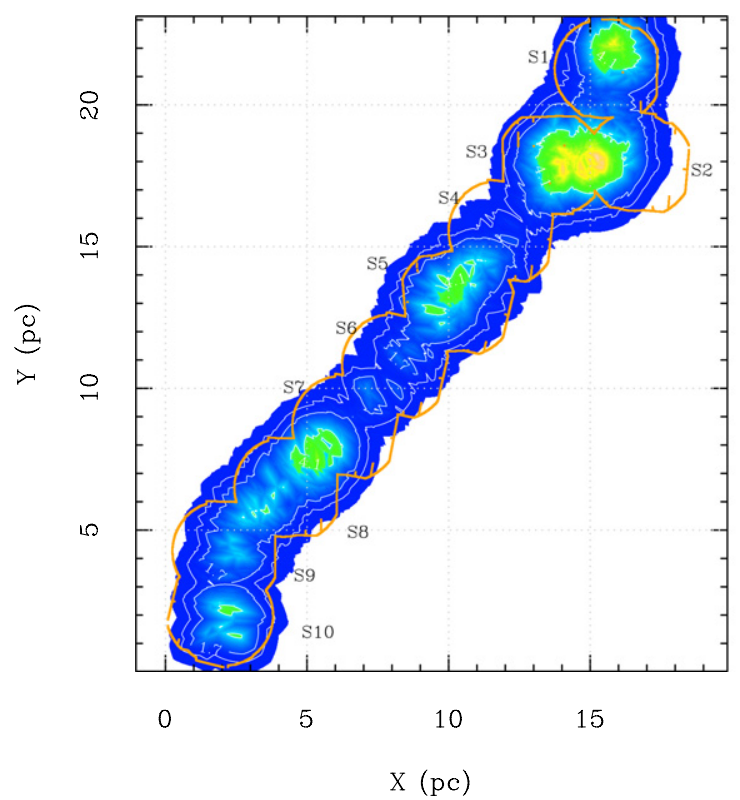

Class III

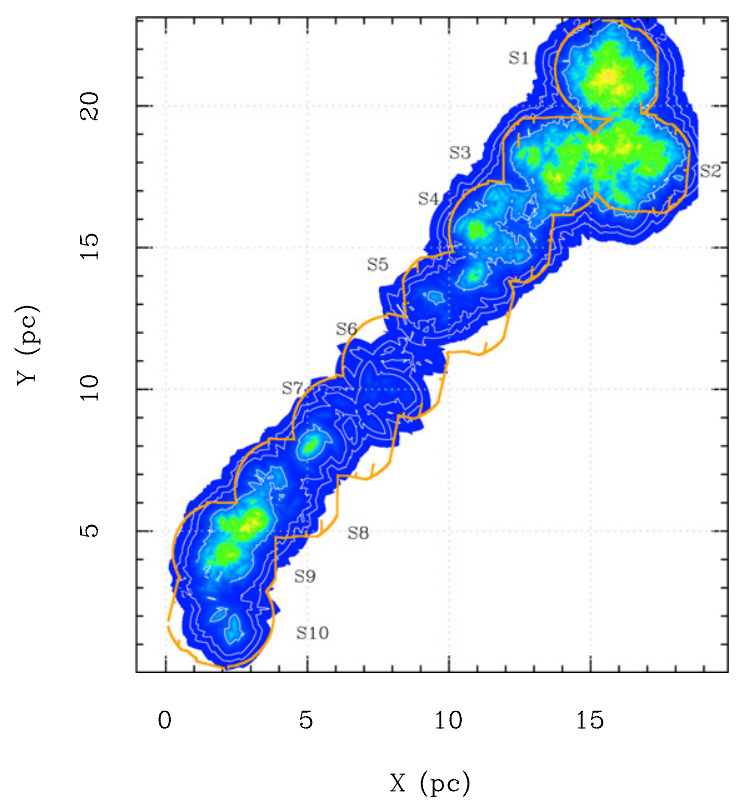

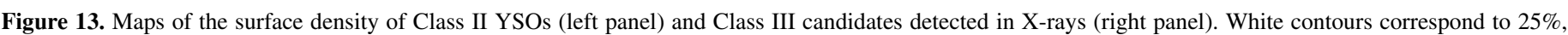

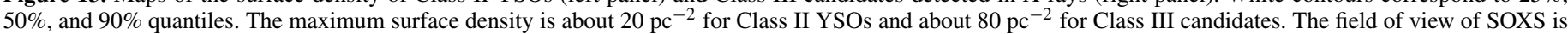

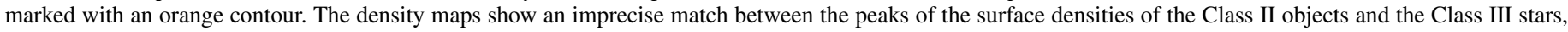

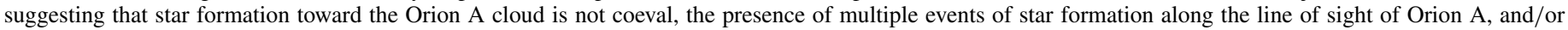
migration of the older stars.

(A color version of this figure is available in the online journal.)

Class III candidates in this regions. In the cores, about $90 \%$ of the Class III and Class II objects have separations $<0.3 \mathrm{pc}$. In comparison, for the entire L1641 region, $90 \%$ of the Class III candidates and Class II objects have separations $<0.4 \mathrm{pc}$. The smaller separations in the two core regions suggest that the young stars are more clustered in these regions. In the $\mathrm{ONC}$, we find evidence of clustering by comparing the separations of the Class II and Class III objects. Although we excluded the densest part across $N 2$ and $N 3$ fields, we find a median separation of $0.2 \mathrm{pc}$ for the Class II objects only. In contrast, the Class III objects in the ONC are more dispersed and have NN distances similar to those in L1641, i.e., median separations of $0.3 \mathrm{pc}$.

While the distributions of NN distances enable us to determine the degree of clustering, the maps of the surface density of stars allow us to evaluate difference in the spatial distributions between Class II objects and Class III stars. Figure 13 shows surface density maps of Class III candidates and Class II objects. For each point in a grid with resolution $\sim 20^{\prime \prime}$, we calculated the distance to the sixth nearest object in the sample of Class II and Class III YSOs. The area of the circle with the radius equal to the sixth object and the number of objects inside that area are used to compute the surface density. We have normalized the maps to the ratio of number of Class III stars to Class II objects. In dealing with three-dimensional (3D) projection effects, these maps and the NN distances analysis are constructed by assuming that the differences along the line of sight are similar to those in the plane of the sky. Effects of 3D projection could be important, and a significant fraction of the stars around $\iota$ Ori can be $100 \mathrm{pc}$ closer than the ONC, as we deduced from a comparison of XLFs. These facts can constitute a bias to the NN distance analysis and to the surface density maps. However, we cannot select the stars belonging to the foreground cluster only, and separate them from the genuine Orion A population, although we know that a large fraction of the $l$ Ori cluster is made up of Class III stars. Given that this cluster is closer than the ONC, it appears to have a higher stellar density than the background stellar population of Orion A.

The Class III objects are denser in the northern part of the survey around ` Ori and sparse in the filament of L1641. In L1641 S, Class III objects are denser across the fields $S 8-S 9$. The density of Class III objects reaches a peak of 80 stars $\mathrm{pc}^{-2}$ in field $S 1$ ( $\iota$ Ori), other local maxima are around $10-15$ stars $\mathrm{pc}^{-2}$. Hence, we can recognize two main groups of Class III objects where their surface density is above $10 \mathrm{pc}^{-2}$ : one in the northern fields near $\iota$ Ori and the other in L1641 $\mathrm{S}$ separated by a very low density region centered on field $S 6$ (Figure 13, right panel). The absence of Class III candidates in the middle part of the L1641 filament is not related to a lower sensitivity of the survey in that region. $S 6$ field has the same exposure time of other fields; here there is a real deficiency of YSOs.

Class II YSOs are most numerous around $\iota$ Ori and merge into the ONC to the north. They are grouped into small subclusters along the filament of L1641. The surface density of Class II objects has a peak of $\sim 20 \mathrm{stars} \mathrm{pc}^{-2}$ in L1641 N (fields $S 2-S 3$ ) and several other local maxima of 4-8 stars pc ${ }^{-2}$ across fields $S 7-S 9$. Class II objects are distributed differently than the Class III candidates. By comparing the two maps (Figure 13), we note that the high-density zones do not overlap exactly. This is most evident in the fields $S 2$ and $S 9$. Class II objects are more concentrated and offset with respect to the centers of the corresponding Class III clusters, suggesting a segregation effect of the YSOs due to their evolutionary stage.

We have calculated the surface density maps for the YSOs in the northern fields $N 1, N 2$, and N3 (Figure 14). Class III 
Class I

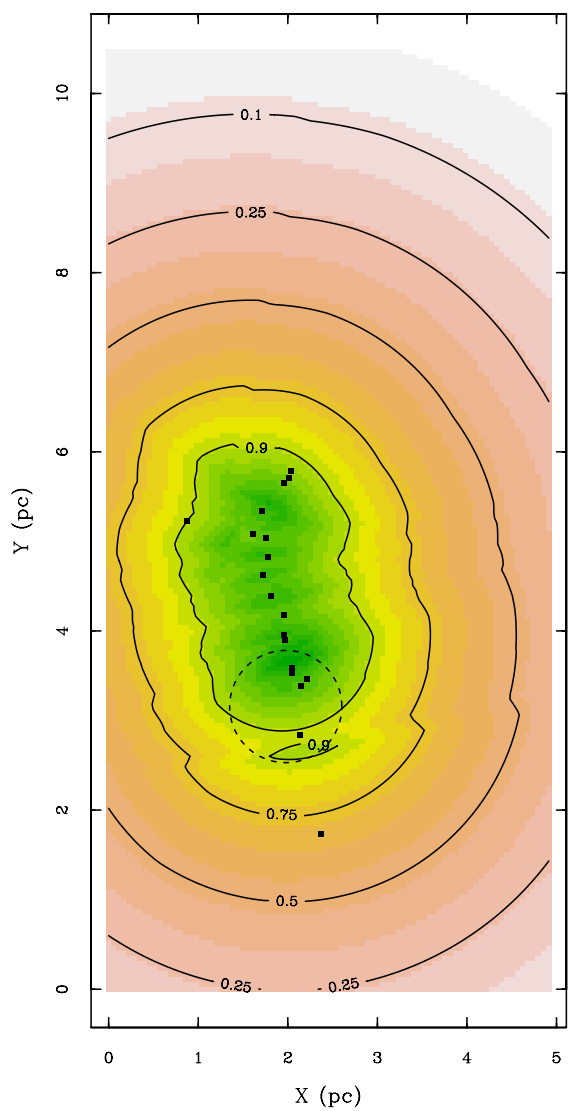

Class II

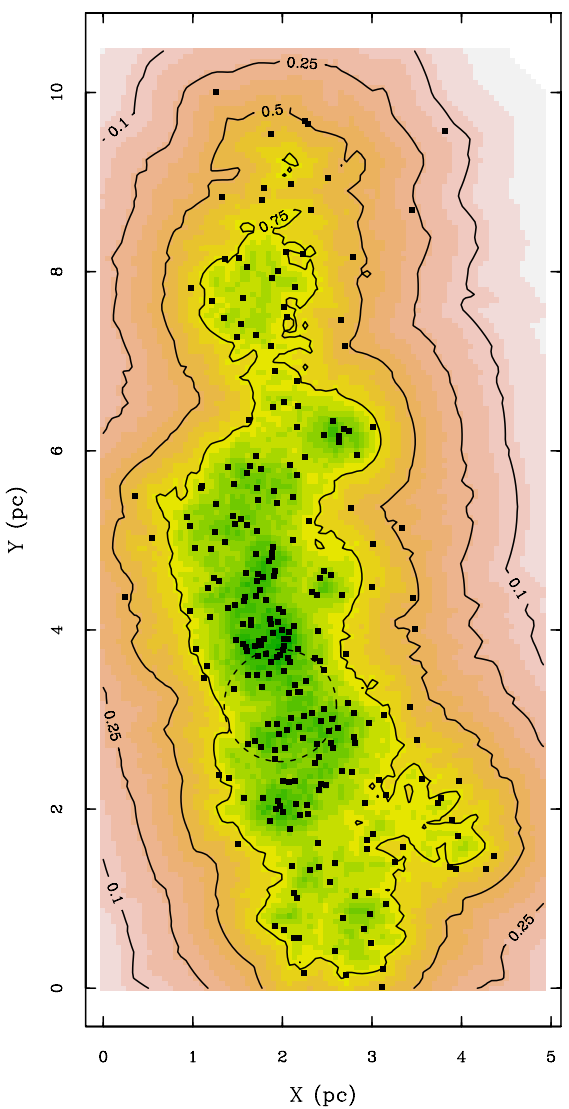

Class III cand.

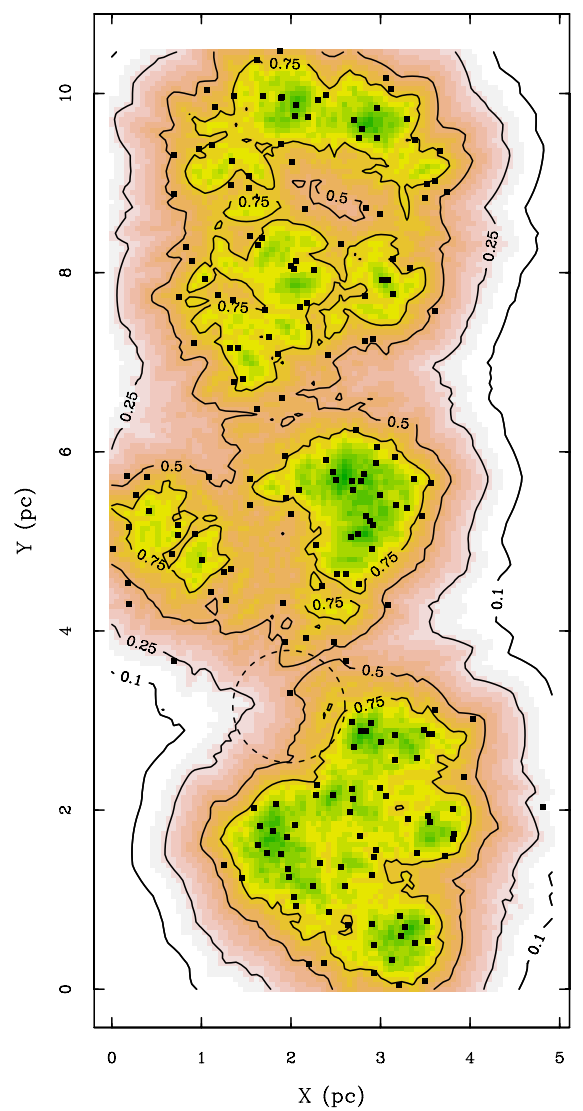

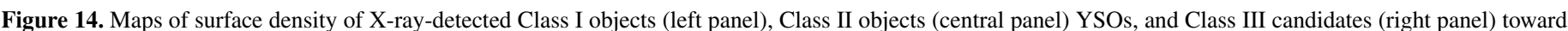

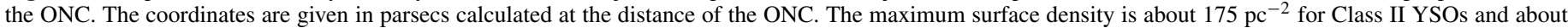

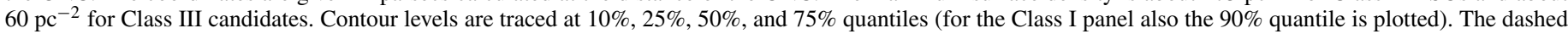

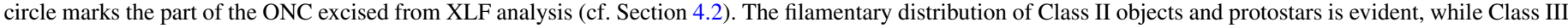
stars are more spread out. Star formation might have proceeded sequentially from outside inward, or the older stars have migrated farther away.

(A color version of this figure is available in the online journal.)

candidates are spread across the image but show a lower density in fields $N 1$ and $N 2$. The latter includes the densest part of the ONC, where strong absorption limits the X-ray sensitivity and bright nebulosity limits the IRAC sensitivity. The low density in $N 2$ is likely due to a real feature in the distribution of Class III stars.

The filamentary shape of the Orion A cloud, as traced by the positions of Class II objects and the few Class I protostars detected in X-rays, is evident. Class I objects detected in Xrays form a chain, most of them lying in the densest part of the cloud. The $75 \%$ contour of the surface density map of Class II objects is about 2 pc wide which is comparable to the Jeans length of a cloud at $T \sim 10-15 \mathrm{~K}$ and a mass of a few dozens of solar masses. It is worth noting the complementary nature of the distributions of Class II and Class III objects which is also observed in L1641. Either they have migrated from their birth place at the center of the filament, or, because of the collapse of the cloud, they formed first in the outer parts and then continued to form stars in the central part of the filament.

\section{IDENTIFICATION OF THREE CLASS III RICH CLUSTERS TOWARD THE ORION A CLOUD}

One of the primary results of the SOXS survey is the discovery of three regions in the Orion A cloud rich in Class III objects. The first of these clusters is toward NGC 1981 in the $N 1$ field
(Figure 9). This field is distinguished by a Class III/Class II ratio of 2.2, as compared to ratios less than 1 for the $N 2$ and $N 3$ fields (Section 4.2). NGC 1981 is found directly north of the Orion A cloud and the NGC 1977 nebula suggesting that it formed from the Orion A cloud and has dispersed the cloud material surrounding the cluster (see Figure 2 in this paper; Figure 1 of Alves \& Bouy 2012). The NGC 1977 cluster appears to be younger than NGC 1981 since it is richer in Class II objects than the latter (Figure 10, Peterson \& Megeath 2008).

The second new cluster of Class III stars is L1641 S located in the $S 9$ and $S 8$ fields toward the southern end of L1641 (Strom et al. 1993; see Figure 11). This cluster has $N_{\mathrm{H}}$ values corresponding to $A_{V}$ values up to $18 \mathrm{mag}$ (Figure 5) and shows an elongation aligned with the L1641 cloud, indicating that this cluster is associated with the cloud. An H-R diagram of this cluster shows an age of $\sim 3 \mathrm{Myr}$, older than that of the other groups in the L1641 cloud (Allen 1995; Allen \& Davis 2008). The Class III/Class II ratio of the $S 9$ field where the cluster is centered, $r \sim 5.5$, is significantly higher than that of the neighboring $S 8$ and $S 10$ fields, 1.0 and 0.87 , respectively; this is evidence that the L1641 S cluster is significantly older than young stars in the nearby regions of L1641.

The third cluster is found toward the O9III+B1III system of $\iota$ Ori and the NGC 1980 cluster (hereafter we refer to this as the $\iota$ Ori cluster). This cluster is identified by Alves \& Bouy (2012; see also Pillitteri et al. 2011; Bally 2008), who noticed 
a concentration of low extinction stars surrounding $\iota$ Ori. On the basis of the low extinction to the stars, they argued that this is a large cluster in the foreground of the Orion A cloud. Our XMM survey confirms their result by revealing an extended cluster of Class III objects with a density peak centered on $\iota$ Ori. The cluster appears to extend through the $S 1, S 2, S 3, S 4$, and $S 5$ fields, more than $1^{\circ}$ on the sky. The morphology and full extent of this cluster cannot be determined from our survey given that it appears to continue beyond our XMM-Newton fields. On the other hand, the Class II objects follow the filamentary structure of the molecular gas and appear to be mostly part of the molecular cloud.

The Class III objects toward ^ Ori have low values of $N_{\mathrm{H}}$ absorption (Figure 5), consistent with the location of this cluster in the foreground respect to the Orion A cloud. As shown in Section 4.1.4, the XLF of the Class III objects toward the $\iota$ Ori cluster is shifted by $\sim 0.3 \pm 0.1$ dex to higher luminosities than that of Class III objects in the southern L1641 fields. This shift is consistent with the presence of a large cluster of Class III objects in the foreground of Orion A. Furthermore, the shift of $0.3 \pm 0.1$ dex implies a distance that is $\sim 1.4$ times closer than the objects in the L1641 fields. If we use the adopted distance of 414 pc for L1641 (Menten et al. 2007) and the ONC, then the distance to the $\iota$ Ori cluster is $\sim 300 \mathrm{pc}$ with a $\sigma$ range of 261-328 pc. In contrast, the XLFs of the Class II objects coincident with the $\iota$ Ori cluster and those in the southern region of L1641 are very similar, indicating that the Class II objects throughout the survey are at a common distance and are associated with the Orion A cloud. We can give an estimate of the number of members in the ${ }_{l}$ Ori cluster. We have 313 Class III stars in fields $S 1-S 4$. If we assume a circular shape and a radius of $\sim 1 \mathrm{deg}(\sim 5.5 \mathrm{pc}$ at $300 \mathrm{pc})$ centered on $\iota$ Ori, we estimate that about 1000 members are found this cluster.

Hoogerwerf et al. (2001) traced the origin of the runaway $\mathrm{O}$ stars $\mu \mathrm{Col}$ and $\mathrm{AE}$ Aur to an ejection event originating at $\iota$ Ori system 2.5 Myr ago. Using Monte Carlo simulations based on the Hipparcos proper motions and parallaxes of these three stars, they determined a distribution of likely distances for the event. Their distribution peaked at $325 \mathrm{pc}$, although it is extended from 250 to 500 pc. Since in 2001 the ONC was the only known nearby cluster, they proposed that the ONC at $\sim 414 \mathrm{pc}$ was the progenitor cluster of $\iota$ Ori and the two runaway stars. On the basis of this hypothetical connection between $\iota$ Ori and the ONC, Tan et al. (2006) argued that the ONC must be a minimum of $2.5 \mathrm{Myr}$ old to have had time to form the four stars participating in this ejection event. However, the detection of this previously unknown cluster around $\iota$ Ori reveals the progenitor of the $\iota$ Ori system. We note that the crude distance estimate we obtained from the XLF, $\sim 300 \mathrm{pc}$, is close to the peak of the distribution of distances determined by Hoogerwerf et al. (2001) for $\iota$ Ori. Furthermore, given that the cluster is primarily traced by Class III objects, we argue that the $\iota$ Ori cluster is $\geqslant 5 \mathrm{Myr}$ old, consistent with the 4-5 Myr age estimated by Alves \& Bouy (2012). This is also consistent with the age of the ejection event of $\mu \mathrm{Col}$ and $\mathrm{AE}$ Aur. The association of the ejection event with the $\iota$ Ori cluster also removes the minimum age constraint of the ONC $(t \geqslant 2.5 \mathrm{Myr})$ given by Tan et al. (2006).

\section{CONCLUSIONS}

In this paper, we have presented the initial results of the SOXS survey designed to explore the young stellar population in the Orion A molecular cloud south of the Orion Nebula, typically referred to as the Lynds 1641 region (L1641). L1641 is a filamentary and highly structured part of the Orion A cloud that extends southeast of the ONC and fills a $3^{\circ} \times 1^{\circ}$ region. The $\mathrm{X}$-ray survey focused on the eastern denser half of the cloud. We have used a multi-band approach for studying the PMS stars and protostars, which were identified either by IR excesses in the Class I and II phases, or by elevated X-ray emission through to the Class III stage. We have described the data analysis and the main properties that we have derived from X-rays observations. Furthermore, we have derived spatial density maps of the young stars and compared their spatial distribution in L1641 to that of the ONC.

We have detected 1060 X-ray sources, with 972 sources having at least one Spitzer counterpart, and about $70 \%$ of these being young stars in evolutionary stages ranging from protostars to diskless young stars. We estimate that there are between 1800 and 2350 young stars in SOXS. We further estimate that there may be $\sim 365$ more young stars when extrapolating to the eastern L1641 fraction surveyed by Spitzer alone. This population of young stars is comparable or larger than the population in the ONC, yet it is dispersed in a larger area and contains very few massive stars.

Through simple 1-T and 2-T APEC absorbed models, we have estimated absorption, plasma temperatures, and fluxes for 232 young stars and protostars. We find that the plasma temperatures depend on Class, with Class I objects (i.e., protostars) being hotter than Class II objects (PMS stars with disks) and Class III candidates (diskless PMS stars). $N_{\mathrm{H}}$ values derived from the $\mathrm{X}$-ray spectra map the gas absorption to the young stars. A gradient of $N_{\mathrm{H}}$ from north to south is observed, with a large number of less absorbed Class III candidates around $\iota$ Ori and in L1641 North, whereas the heavily absorbed Class I/II YSOs are located primarily near the southern and the central parts of L1641. The $N_{\mathrm{H}} / A_{K}$ ratio for L1641 is found to be lower than the average value found in the ISM or high-mass starforming regions, but consistent with what is found in NGC 1333 and Serpens. While high-mass star-forming regions, such as RCW 108 and RCW 38, show ISM-like $N_{\mathrm{H}} / A_{K}$ ratio, here we find less gas absorption with respect to dust extinction than typically seen in the ISM. An explanation for this behavior is that low-mass star-forming regions lack intense radiation fields typical of high-mass star-forming regions. We speculate that the ambient radiation field may influence the coagulation and the sizes of dust grains and thereby the $N_{\mathrm{H}} / A_{K}$ ratios.

We have analyzed the spatial distribution of the PMS stars and protostars. Class I and II objects appear clustered into small groups in the L1641 North and South regions. The Class III candidates are in contrast more dispersed, with two main groups around $\iota$ Ori and in L1641 S. In the central fields of L1641, Class III stars are less common. We suggest that the Class III objects preferentially trace an older population of PMS stars toward L1641. Strong evidence for this older population is found in a cluster of Class III stars around the massive B0III $\iota$ Ori (the southernmost star of Orion's sword) and NGC 1980. The high concentration of Class III stars in this region further confirms that the cluster of low extinction stars found by Alves $\&$ Bouy (2012) around the $\iota$ Ori cloud is a cluster of PMS stars that formed prior to the ONC. We also find a concentration of Class III objects toward L1641 S; this is the first evidence for a cluster of older stars in the L641 dark cloud itself.

We have compared the XLFs of Class II objects and Class III stars for which the membership has been determined on the basis of combined criteria of IR excess (for Class II objects), X-ray emission (for Class III stars), and/or the presence of spectral 
features such as Li doublet absorption or $\mathrm{H} \alpha$ emission (Hsu et al. 2012). The XLFs of Class III stars reveal that the group of stars in L1641 N and around ८ Ori appears more luminous than those in L1641 S. We ascribe this to a closer distance of $300 \mathrm{pc}$ for the cluster of stars surrounding $\iota$ Ori. These findings indicate that the cluster of stars of which $\iota$ Ori is the most massive remaining member formed in the foreground of the ONC. Furthermore, the cluster surrounding $\iota$ Ori appears to be the site of the proposed ejection of the runaway $\mathrm{O}$ stars $\mu \mathrm{Col}$ and $\mathrm{AE}$ Aur $\geqslant 2.5$ Myr ago.

In addition we have analyzed three archival XMM fields covering the northern Orion A cloud. The X-ray sources in this northern region follow the narrow filamentary structure of the ONC. Class II and Class I objects are detected along the cloud filament, while Class III candidates are spread out along the peripheral borders of the filament. We suggest that the Class III candidates are more spread out because they have had time to drift away, or that the they formed by a sequence of star formation progressing inward toward the filament. We also find a Class III rich cluster of young stars toward NGC 1981; this appears to be the result of a previous star formation event directly north of the Orion A cloud. In total, the SOXS survey has revealed the non-coeval nature of the star formation toward Orion A. We find not only further evidence for a foreground cluster surrounding $\iota$ Ori, but also more evolved regions of recent star formation at both the northern and southern ends of the Orion A cloud. This non-coevality is an important constraint on models for the formation and evolution of the Orion A cloud.

The XMM-Newton guest investigator program supported I.P. through grant NNX09AP46G. S.J.W. was supported by NASA contract NAS8-03060 to the Chandra Science Center. This work is based on observations obtained with XMM-Newton, an ESA science mission with instruments and contributions directly funded by ESA Member States and NASA, and in part on observations made with the Spitzer Space Telescope, which is operated by the Jet Propulsion Laboratory, California Institute of Technology under a contract with NASA. This publication makes use of data products from the Two Micron All Sky Survey, which is a joint project of the University of Massachusetts and the Infrared Processing and Analysis Center/California Institute of Technology, funded by the National Aeronautics and Space Administration and the National Science Foundation.

Facilities: XMM (EPIC), Spitzer (IRAC, MIPS).

\section{REFERENCES}

Allen, L., Megeath, S. T., Gutermuth, R., et al. 2007, in Protostars and Planets V, ed. B. Reipurth, D. Jewitt, \& K. Keil (Tucson, AZ: Univ. Arizona Press), 361

Allen, L. E. 1995, Star Formation in Lynds 1641 (Amherst, MA: Univ. Massachusetts)

Allen, L. E., Calvet, N., D'Alessio, P., et al. 2004, ApJS, 154, 363

Allen, L. E., \& Davis, C. J. 2008, in Handbook of Star Forming Regions, Vol. I: The Northern Sky ASP Monograph Publications, Vol. 4, ed. B. Reipurth (San Francisco, CA: ASP), 621

Alves, J., \& Bouy, H. 2012, A\&A, 547, A97

Argiroffi, C., Flaccomio, E., Bouvier, J., et al. 2011, A\&A, 530, A1

Arnaud, K., Dorman, B., \& Gordon, C. 1999, ascl soft, record ascl:9910.005, 10005

Bally, J. 2008, in Handbook of Star Forming Regions, Vol. I: The Northern Sky ASP Monograph Publications, Vol. 4, ed. B. Reipurth (San Francisco, CA: ASP), 459

Bally, J., Lanber, W. D., Stark, A. A., \& Wilson, R. W. 1987, ApJL, 312, L45
Baraffe, I., Chabrier, G., Allard, F., \& Hauschildt, P. H. 1998, A\&A, 337, 403 Brickhouse, N. S., Cranmer, S. R., Dupree, A. K., Luna, G. J. M., \& Wolk, S. 2010, ApJ, 710, 1835

Buckle, J. V., Davis, C. J., Francesco, J. D., et al. 2012, MNRAS, 422, 521

Carpenter, J. M. 2000, AJ, 120, 3139

Chapman, N. L., Mundy, L. G., Lai, S.-P., \& Evans, N. J., II. 2009, ApJ, 690, 496

Chiar, J. E., Ennico, K., Pendleton, Y. J., et al. 2007, ApJL, 666, L73

Damiani, F., Flaccomio, E., Micela, G., et al. 2003, ApJ, 588, 1009

Damiani, F., Maggio, A., Micela, G., \& Sciortino, S. 1997, ApJ, 483, 370

Fang, M., van Boekel, R., Wang, W., et al. 2009, A\&A, 504, 461

Favata, F., Flaccomio, E., Reale, F., et al. 2005, ApJS, 160, 469

Favata, F., \& Micela, G. 2003, SSRv, 108, 577

Feigelson, E. D., Getman, K., Townsley, L., et al. 2005, ApJS, 160, 379

Feigelson, E. D., \& Montmerle, T. 1999, ARA\&A, 37, 363

Flaherty, K. M., Pipher, J. L., Megeath, S. T., et al. 2007, ApJ, 663, 1069

Getman, K. V., Feigelson, E. D., Townsley, L., et al. 2006, ApJS, 163, 306

Getman, K. V., Flaccomio, E., Broos, P. S., et al. 2005, ApJS, 160, 319

Gilli, R., Comastri, A., \& Hasinger, G. 2007, A\&A, 463, 79

Gorenstein, P. 1975, ApJ, 198, 95

Guarcello, M. G., Micela, G., Peres, G., Prisinzano, L., \& Sciortino, S. 2010, A\&A, 521, A61

Güdel, M., Skinner, S. L., Audard, M., Briggs, K. R., \& Cabrit, S. 2008, A\&A, 478, 797

Günther, H. M., \& Schmitt, J. H. M. M. 2008, A\&A, 481, 735

Günther, H. M., Wolk, S. J., Spitzbart, B., et al. 2012, AJ, 144, 101

Gutermuth, R. A., Bourke, T. L., Allen, L. E., et al. 2008, ApJL, 673, L151

Gutermuth, R. A., Megeath, S. T., Myers, P. C., et al. 2009, ApJS, 184, 18

Gutermuth, R. A., Megeath, S. T., Pipher, J. L., et al. 2005, ApJ, 632, 397

Gutermuth, R. A., Pipher, J. L., Megeath, S. T., et al. 2011, ApJ, 739, 84

Hoogerwerf, R., de Bruijne, J. H. J., \& de Zeeuw, P. T. 2001, A\&A, 365, 49

Hsu, W.-H., Hartmann, L., Allen, L., et al. 2012, ApJ, 752, 59

Hsu, W.-H., Hartmann, L., Allen, L., et al. 2013, ApJ, 764, 114

Kenyon, S. J., \& Hartmann, L. 1995, ApJS, 101, 117

Kryukova, E., Megeath, S. T., Gutermuth, R. A., et al. 2012, AJ, 144, 31

Kuhn, M. A., Getman, K. V., Feigelson, E. D., et al. 2010, ApJ, 725, 2485

Lada, C. J., \& Adams, F. C. 1992, ApJ, 393, 278

Lada, C. J., Lada, E. A., Clemens, D. P., \& Bally, J. 1994, ApJ, 429, 694

Lavalley, M. P., Isobe, T., \& Feigelson, E. D. 1992, BAAS, 24, 839

Lombardi, M., \& Alves, J. 2001, A\&A, 377, 1023

Luhman, K. L. 1999, ApJ, 525, 466

McClure, M. 2009, ApJL, 693, L81

Megeath, S. T., Allen, L. E., Gutermuth, R. A., et al. 2004, ApJS, 154, 367

Megeath, S. T., Gutermuth, R., Muzerolle, J., et al. 2012, AJ, 144, 192

Menten, K. M., Reid, M. J., Forbrich, J., \& Brunthaler, A. 2007, A\&A, 474, 515

O'Dell, C. R. 2001, ARA\&A, 39, 99

Ormel, C. W., Min, M., Tielens, A. G. G. M., Dominik, C., \& Paszun, D. 2011, A\&A, 532, A43

Peterson, D. E., \& Megeath, S. T. 2008, in Handbook of Star Forming Regions, Vol. I: The Northern Sky ASP Monograph Publications, Vol. 4, ed. B. Reipurth (San Francisco, CA: ASP), 590

Pillitteri, I., Sciortino, S., Flaccomio, E., et al. 2010, A\&A, 519, A34

Pillitteri, I., Wolk, S. J., Allen, L., et al. 2011, in ASP Conf. Ser. 448, 16th Cambridge Workshop on Cool Stars, Stellar Systems, and the Sun, ed. C. Johns-Krull, M. K. Browning, \& A. A. West (San Francisco, CA: ASP), 705 Preibisch, T., Kim, Y.-C., Favata, F., et al. 2005, ApJS, 160, 401

Prisinzano, L., Micela, G., Flaccomio, E., et al. 2008, ApJ, 677, 401 Puccetti, S., Vignali, C., Cappelluti, N., et al. 2009, ApJS, 185, 586 Rieke, G. H., \& Lebofsky, M. J. 1986, ApJ, 304, 326

Rieke, G. H., Young, E. T., Engelbracht, C. W., et al. 2004, ApJS, 154, 25

Roy, A., Martin, P. G., Polychroni, D., et al. 2013, ApJ, 763, 55

Sciortino, S., Micela, G., Damiani, F., et al. 2001, A\&A, 365, L259

Sherry, W. H., Walter, F. M., \& Wolk, S. J. 2004, AJ, 128, 2316

Smith, R. K., Brickhouse, N. S., Raymond, J. C., \& Liedahl, D. A. 1999, BAAS, 31,735

Strom, K. M., Margulis, M., \& Strom, S. E. 1989, ApJL, 345, L79

Strom, K. M., Strom, S. E., \& Merrill, K. M. 1993, ApJ, 412, 233

Strom, K. M., Strom, S. E., Wilkin, F. P., et al. 1990, ApJ, 362, 168

Tan, J. C., Krumholz, M. R., \& McKee, C. F. 2006, ApJL, 641, L121

Vuong, M. H., Montmerle, T., Grosso, N., et al. 2003, A\&A, 408, 581

Wang, J., Townsley, L. K., Feigelson, E. D., et al. 2008, ApJ, 675, 464

Winston, E., Megeath, S. T., Wolk, S. J., et al. 2010, AJ, 140, 266

Wolk, S. J., Spitzbart, B. D., Bourke, T. L., \& Alves, J. 2006, AJ, 132, 1100

Wolk, S. J., Spitzbart, B. D., Bourke, T. L., et al. 2008, AJ, 135, 693

Wright, N. J., Drake, J. J., Drew, J. E., et al. 2012, ApJL, 746, L21 